This article is licensed under the Creative Commons Attribution-NonCommercial-NoDerivatives 4.0 Interna-

\title{
Therapeutic Potential of Gamma-
} Irradiated Resveratrol in Ulcerative Colitis via the Anti-Inflammatory Activity and Differentiation of Tolerogenic Dendritic Cells

\author{
Woo Sik Kim ${ }^{a} \quad H a-Y e o n$ Song ${ }^{a}$ Sajid Mushtaq ${ }^{a} \quad$ Jin-Man Kim ${ }^{b}$ Eui-Hong Byun ${ }^{c}$ \\ Jae-Min Yuk d,e Eui-Baek Byun ${ }^{a}$ \\ ${ }^{a}$ Advanced Radiation Technology Institute, Korea Atomic Energy Research Institute, Jeongeup, Republic \\ of Korea, 'Department of Pathology, College of Medicine, Chungnam National University, Daejeon, \\ Republic of Korea, 'Department of Food Science and Technology, Kongju National University, Gongju, \\ Republic of Korea, dDepartment of Infection Biology, College of Medicine, Chungnam National \\ University, Daejeon, Republic of Korea, eDepartment of Medical Science, College of Medicine, \\ Chungnam National University, Daejeon, Republic of Korea
}

\section{Key Words}

Resveratrol • Gamma irradiation • Tolerance $\cdot$ Anti-inflammatory activity • Inflammatory bowel disease

\begin{abstract}
Background/Aims: New therapeutic strategies and the development of treatments against inflammatory bowel disease (IBD) require the initiation of immune tolerance and inhibition of excessive inflammation. Resveratrol, a polyphenolic compound, is a powerful immunosuppressor, but it can lead to apoptotic death of normal cells at high concentrations. When we induced a structural modification of resveratrol by gamma irradiation, we were able to investigate the potential tolerogenic and anti-inflammatory effect of a new radiolysis product (named $\gamma$-Res) during dendritic cell (DC) activation/differentiation. Methods: The potential tolerogenic and anti-inflammatory effect of $\gamma$-Res were investigated by cytokine secretion, surface molecule expression, antigen uptake ability, antigen presenting ability, signaling pathway, and mixed lymphocyte reaction (MLR) assay using enzyme-linked immunosorbent assay (ELISA), western blot and flow cytometry. Results: LPS-activated DCs treated with $\gamma$-Res exhibited alterations in their mature and functional statuses including a strongly inhibited cytokine production, surface molecule expression, antigen-presenting ability, and activated DC-induced T cell proliferation/activation. In addition, the DCs generated by the

WS Kim and HY Song contributed equally to this work.




\section{Cellular Physiology Cell Physiol Biochem 2019;52:1117-1138

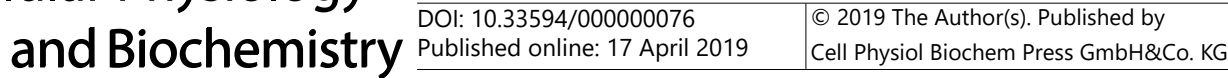 \\ Kim et al.: Gamma-Irradiated Resveratrol Induces Tolerance and Anti-Inflammatory \\ Activity in Dendritic Cells}

$\gamma$-Res treatment during DC differentiation induced a decreased surface molecule expression and increased IL-10 production without altering the levels of TNF- $\alpha$ and IL-12p70, thereby promoting the inhibition of $T$ cell proliferation/activation and the induction of regulatory $T$ cells via interaction with DCs in vitro. Furthermore, in the in vivo DSS-induced colitis model, $\gamma$-Res treatment conferred protective immunity with a decrease in IFN- $\gamma^{+} \mathrm{CD} 4^{+}$and IL$17 \mathrm{~A}^{+} \mathrm{CD} 4^{+} \mathrm{T}$ cells and imparted protection by reducing the disease activity and histological disease score and increasing the survival rate in dextran sulfate sodium (DSS)-induced colitis in mice. Conclusion: Thus, our results suggest that $\gamma$-Res may be an excellent candidate for use in IBD treatment.

(C) 2019 The Author(s). Published by Cell Physiol Biochem Press GmbH\&Co. KG

\section{Introduction}

The main cause of inflammatory bowel disease (IBD) including Crohn's disease (CD) and ulcerative colitis (UC) is not yet fully understood; however, we do know that this disease is the result of an abnormal immune response triggered by specific genetic and/ or immunologic defects in the gastrointestinal tract [1]. Importantly, the immune systems involved are influenced by the dysfunction of the mucosal innate and adaptive immune cells, and these cells recognize foreign antigens from food and intestinal bacteria leading to a chronic inflammation response $[1,2]$. In CD pathogenesis, IFN- $\gamma$, TNF- $\alpha$, and IL-17 cytokines are mainly induced by increased intestinal expression in Th1 and Th17 cells, which may contribute to severe intestinal damage by immune cell activation and expression of apoptosis-related proteins that inhibit the expression of anti-apoptotic proteins [3-5]. On the other hand, UC is usually viewed as Th2 immunity because of the excessive expression of the principal Th2 effector cytokines (IL-5 and IL-13) and TNF- $\alpha$ [5], and these responses augment the ulceration and inflammation by B cell activation, activation/recruitment of eosinophils and NKT-cell-mediated cytotoxicity [6, 7]. In this regard, to solve the problems of the immune response associated with IBD, many studies have focused on effective regulation for imbalanced cytokines and immune cell populations contributing to the immunopathogenesis of IBD.

Recently, polyphenolic compounds, including resveratrol, epigallocatechin gallate (EGCG), curcumin, quercetin, and rutin, have been reported as potential candidates to help IBD. These compounds are known to interact with various immune cells. They have been proven effective in reducing excessive inflammation by regulating inflammatory mediators such as TNF- $\alpha$, IFN- $\gamma$, IL-12, inducible nitric oxide synthase (iNOS), mitogen-activated protein (MAPK), and nuclear factor $\kappa$ B (NF- $\kappa B$ ) for immune cells in IBD models [8-11]. However, most polyphenols may lead to apoptosis of normal cells when used at higher concentrations [12-14], indicating that improved treatment strategies are urgently needed.

Irradiation technology is a useful tool for improving the function of polyphenols via structural modification [15-17]. For example, certain gamma-irradiated polyphenols, such as resveratrol, and luteolin, have been verified as enhancing bioactivity, such as antiinflammatory activity and anti-melanogenic effect, and decreasing the toxicity of the parent compounds $[15,16]$. However, among the gamma-irradiated polyphenols, there is still a lack of knowledge about resveratrol; it is unclear how its structure changes and whether it is involved in protection or immune evasion in the immune systems contributing to IBD.

To understand the functional roles of resveratrol transformed by gamma irradiation in the maturation and differentiation of dendritic cells (DCs) that cause inflammation in IBD, we looked at the anti-inflammatory activity of a new product of resveratrol transformed by gamma irradiation and the phenotypic and functional change induced by the treatment with the new product during DC differentiation as well as the therapeutic effect and immunological contribution caused by the treatment with this new product in an animal model of IBD. 


\section{Cellular Physiology Cell Physiol Biochem 2019;52:1117-1138 \begin{tabular}{l|l}
\hline DOI: 10.33594/000000076 & (c) 2019 The Author(s). Published by
\end{tabular} and BIOChemistry $\frac{\text { Published online: } 17 \text { April } 2019 \quad \text { Cell Physiol Biochem Press GmbH\&Co. KG }}{\text { Kim et al.: Gamma-Irradiated Resveratrol Induces Tolerance and Anti-Inflammatory }}$ \\ Activity in Dendritic Cells}

\section{Materials and Methods}

\section{Mice}

Specific Pathogen Free mice (female C57BL/6 (H-2Kb and I-Ab) and BALB/c (H-2Kd and I-Ad), seven weeks of age) were used in the in vitro and in vivo experiments. These mice were purchased from Orient Bio Inc. (Seoul, Korea). All the mice were acclimated to the following controlled conditions: temperature $(25 \pm$ $2{ }^{\circ} \mathrm{C}$ ), humidity $(55 \pm 5 \%$ ), and $12 \mathrm{~h}$ light/dark cycle at the Central Animal Research Laboratory at the Korea Atomic Energy Research Institute (KAERI, Jeongeup, Korea). Animal experimental protocols were approved by the Institutional Animal Care and Use Committee of KAERI (KAERI-IACUC-2017-034) and performed in strict compliance with the guidelines prescribed by the committee.

\section{Preparation of gamma-irradiated resveratrol}

Resveratrol was purchased from R\&D Systems (Minneapolis, MN, USA). The resveratrol solution (1 $\mathrm{mg} / \mathrm{mL}$ ) dissolved in methanol was irradiated at a dose of $50 \mathrm{kGy}(10 \mathrm{kGy} / \mathrm{h}$ ) by a cobalt-60 irradiator (point source AECL, IR-221, MDS Nordion International Co., Ltd., Ottawa, ON, Canada). The methanol was evaporated by a rotary evaporator (Tokyo Rikakikai Co., Ltd., Japan), and the irradiated resveratrol was stored at $-80^{\circ} \mathrm{C}$.

\section{HPLC analysis and identification of the gamma-irradiated resveratrol}

For analysis of intact resveratrol (intact-Res) and gamma-irradiated resveratrol ( $\gamma$-Res), a $20 \mu \mathrm{L}$ sample was injected into an Agilent HPLC 1260 system (Agilent Technologies, Inc., Santa Clara, CA, USA) with a diode array detector. The chromatographic separation was performed using an Agilent Eclipse XDB-C18 column (4.6 mm $\times 250 \mathrm{~mm}$ i.d., $5 \mu \mathrm{m}$, Agilent Technologies). The mobile phase consisted of $0.1 \%$ formic acid in water (A) and $0.1 \%$ formic acid in acetonitrile (B). The analysis was conducted using an isocratic elution during $50 \mathrm{~min}$. with A-B (75-25). For isolation of the $\gamma$-Res, purification was performed by preparative HPLC (1260 Infinity System, Agilent Technologies). The Agilent Eclipse XDB-C18 column (21.2 $\mathrm{mm} \times 150 \mathrm{~mm}$ i.d., $7.5 \mu \mathrm{m}$ ) was used to isolate the $\gamma$-Res. Identification of the isolated $\gamma$-Res was carried out with an electrospray ionization mass spectrometer (ESI-MS), ${ }^{1} \mathrm{H}$-nuclear magnetic resonance (NMR), and ${ }^{13} \mathrm{C}$-NMR. The NMR spectra were recorded with a Bruker $500 \mathrm{MHz}$ spectrometer $\left({ }^{1} \mathrm{H}, 500 \mathrm{MHz}\right.$ and ${ }^{13} \mathrm{C}, 125$ $\mathrm{MHz}$ ) using DMSO- $d_{6}$.

\section{Generation of BMDCs and gamma-irradiated resveratrol-treated DCs}

To generate bone-marrow-derived dendritic cells (BMDCs), intact-Res-treated DCs (intact-Res-DCs) and $\gamma$-Res-treated DCs ( $\gamma$-Res-DCs), whole bone marrow cells from C57BL/ 6 mice were lysed of red blood cells (RBCs) using RBC lysing buffer (Sigma-Aldrich), and then, the lysed cells were maintained in 6-well plates $\left(1 \times 10^{6}\right.$ cells $/ \mathrm{ml} ; 2 \mathrm{~mL} /$ well $)$ and cultured at $37^{\circ} \mathrm{C}$ in the presence of $5 \% \mathrm{CO}_{2}$, using complete-RPMI 1640 (c-RPMI 1640, GIBCO, Carlsbad, CA, USA) containing 10\% FBS (GIBCO), $100 \mathrm{U} / \mathrm{mL}$ penicillin, $100 \mathrm{U} / \mathrm{mL}$ streptomycin, $20 \mathrm{ng} / \mathrm{mL}$ GM-CSF (JW CreaGene, Daegu, Korea) and $0.5 \mathrm{ng} / \mathrm{mL}$ IL-4 (JW CreaGene). On day three of the culture, $1 \mathrm{~mL}$ c-RPMI 1640 medium was added to each well of the BMDC differentiation plates, and $1 \mathrm{~mL}$ c-RPMI 1640 medium was added to each well of the intact-Res-DCs and $\gamma$-Res-DC differentiation plates along with $\gamma$-Res and intact-Res at a concentration of 15 and $50 \mu \mathrm{g} / \mathrm{mL}$. On day six of the culture, the plate media were changed with $1 \mathrm{~mL}$ c-RPMI 1640 medium per well. On day eight of the culture, the cells were harvested and stained with fluorescein-conjugated CD11c mAb. Over $90 \%$ of the cells expressed CD11c.

\section{Cytotoxicity and cell viability}

To analyze cytotoxicity, after 8 days of culturing, the BMDCs, intact-Res-DCs and $\gamma$-Res-DCs were stained with the propidium iodide (PI; BD Bioscience, San Diego, CA, USA) method. Additionally, the cultured BMDCs were seeded in 48-well plates at a density of $2 \times 10^{5}$ cells/well and treated with the following stimuli for $24 \mathrm{~h}$ : DMSO (Con), LPS (100 ng/mL; Invivogen, San Diego, CA), LPS and intact-Res (30 $\mathrm{mg} / \mathrm{mL})$, or LPS and $\gamma$-Res $(30 \mu \mathrm{g} / \mathrm{mL})$. The cells were washed with PBS and stained with the Annexin V/PI apoptosis detection kit (BD Bioscience) following the manufacturer's instructions. To analyze viability, each group was treated with $10 \mu \mathrm{L}$ EZ-Cytox kit reagent (Daeil Laboratories, Seoul, Korea), and the cells were incubated for $1 \mathrm{~h}$. The absorbance was measured at $450 \mathrm{~nm}$ using an automated micro-plate reader (Zenyth 3100; Anthos Labtec Instruments $\mathrm{GmbH}$, Salzburg, Austria). 


\section{Cellular Physiology Cell Physiol Biochem 2019;52:1117-1138 \\ \begin{tabular}{l|l}
\hline DOI: 10.33594/000000076 & ( 2019 The Author(s). Published by
\end{tabular} \\ Published online: 17 April $2019 \quad$ Cell Physiol Biochem Press GmbH\&Co. KG \\ Kim et al.: Gamma-Irradiated Resveratrol Induces Tolerance and Anti-Inflammatory \\ Activity in Dendritic Cells}

Analysis of the surface molecules on DCs

To analyze the difference in the surface molecule expression of the BMDCs and $\gamma$-Res-DCs, cells were stimulated with LPS for $24 \mathrm{~h}$. Additionally, to analyze the effect of the $\gamma$-Res treatment on the expression of surface molecules induced by the LPS-treated BMDCs, BMDCs were treated with the following stimuli for $24 \mathrm{~h}$ : DMSO (Con), LPS, or LPS and $\gamma$-Res, and then, the cells were stained with anti-CD80, anti-CD86, antiMHC-I, and anti-MHC-II for $20 \mathrm{~min}$. at room temperature (RT). The resulting fluorescence was collected on a FACSverse flow cytometer (BD Biosciences) and analyzed using the FlowJo software (BD Biosciences). Antibodies (Abs) were purchased from BD Biosciences.

\section{Intracellular cytokine staining}

After incubating with GolgiPlug for $9 \mathrm{~h}$, cells were stained with a Live/Dead cell staining kit (Invitrogen, Life Technologies, Naerum, Denmark) using DC and T cell phenotypic markers (anti-CD11c, anti-CD3, antiCD4, and anti-CD8 Abs) for $20 \mathrm{~min}$. at RT and then fixed and permeabilized with a Cytofix/Cytoperm kit (BD Bioscience) following the manufacturer's instructions. Next, anti-TNF-a, anti-IL-12p70, anti-IFN- $\gamma$, anti-IL17A, anti-IL-5, and anti-IL-10 Abs were stained with fluorescein-conjugated secondary Abs in a permeation buffer. The Abs for the phenotypic markers and cytokines of the DCs and T cells were purchased from BD Bioscience.

\section{Cytokine measurements}

The levels of TNF- $\alpha$, IL-12p70, IFN- $\gamma$, IL-2, and IL-10 in the supernatants were measured with an ELISA kit (BD Bioscience) according to the manufacturer's instructions.

\section{Antigen-uptake capacity}

After stimulation with or without LPS, BMDCs and $\gamma$-Res-DCs were cultured in the presence of $1 \mathrm{mg} /$ $\mathrm{ml}$ fluorescein-conjugated dextran (40,000 Da, Sigma-Aldrich) at $37^{\circ} \mathrm{C}$. After $30 \mathrm{~min}$. of treatment, cells were washed with cold PBS and stained using anti-CD11c or DAPI and analyzed by FACSverse or LSM 800 confocal laser scanning microscope (Zeiss, Germany). Images were acquired with the LSM 800 software and processed with a LSM imager.

\section{Isolation of $T$ cells in splenocytes}

For $\mathrm{CD}^{+} \mathrm{T}$ cell isolation in splenocytes, cells isolated from the mice of the DSS-induced colitis model or naïve mice (BALB/C mice) were labeled with anti-CD16/32 and bead-conjugated anti-CD11c Abs (CD11c MicroBeads) or anti-CD3e mAbs (CD3e MicroBeads), respectively, and separated by sequential passage through LS MACS Columns. The purity of the selected $\mathrm{CD}^{+} \mathrm{T}$ cell fractions was $>80 \%$. The columns and antibodies for cell isolation were purchased from Miltenyi Biotec (San Diego, CA).

T cell suppression assay and mixed lymphocyte reaction (MLR)

For the $\mathrm{T}$ cell suppression assay using allogeneic mice, $\mathrm{CD}^{+} \mathrm{T}$ cells separated by the MACS system were isolated from the spleens of the BALB/C mice, stained with CFSE $(5 \mu \mathrm{M})$ at $37{ }^{\circ} \mathrm{C}$, and then washed with PBS (supplemented with 2\% FBS) after $15 \mathrm{~min}$. Next, 48-well plates were coated with anti-CD3 mAb (0.5 $\mu \mathrm{g} /$ $\mathrm{mL})$ and anti-CD28 $\mathrm{mAb}(0.5 \mu \mathrm{g} / \mathrm{mL})$ at $4{ }^{\circ} \mathrm{C}$. After $24 \mathrm{~h}, 5 \times 10^{5} \mathrm{~T}$ cells stained with CFSE were cultured onto plates coated with anti-CD3/CD28 mAbs. In addition, BMDCs and $\gamma$-Res-DCs generated from the C57BL/6 were stimulated with or without LPS for $2 \mathrm{~h}$ and washed with PBS. The ratio between DCs and T cells was $0.5: 1$.

For the MLR assay, BMDCs (from C57BL/6) were treated with LPS in the presence and absence of $\gamma$-Res for $24 \mathrm{~h}$ and then washed and co-cultured with CFSE-labeled T cells (from BALB/C) at a DC to T cell ratio of 0.5:1. After 3 days of co-culturing, each of the cells from the $\mathrm{T}$ cell suppression and MLR experiment was harvested and stained with anti-CD4 and anti-CD8 mAbs. Data were collected on a FACSverse and analyzed with the FlowJo software. The culture supernatants were harvested, and cytokine production was analyzed by ELISA.

\section{Regulatory T cell analysis}

$\mathrm{CD}^{+} \mathrm{T}$ cells were isolated from the spleens of BALB/C mice with the Miltenyi $\mathrm{CD} 4{ }^{+} \mathrm{T}$ cell isolation kit. Isolated $\mathrm{CD}^{+} \mathrm{T}$ cells $\left(5 \times 10^{5}\right.$ cells) were co-cultured with BMDC and $\gamma$-Res-DCs at a variable ratio (DC and 


\section{Cellular Physiology Cell Physiol Biochem 2019;52:1117-1138 \\ \begin{tabular}{ll|l} 
DOI: 10.33594/000000076 & (c)19 The Author(s). Published by
\end{tabular} \\ \begin{tabular}{l|l} 
Published online: 17 April 2019 & Cell Physiol Biochem Press GmbH\&Co. KG \\
\hline
\end{tabular} \\ Kim et al.: Gamma-Irradiated Resveratrol Induces Tolerance and Anti-Inflammatory \\ Activity in Dendritic Cells}

T cell, $0.5: 1$ ) for $72 \mathrm{~h}$ in a 48 -well plate. After $72 \mathrm{~h}$, the cells were harvested and stained with anti-CD3 and anti-CD4 for $30 \mathrm{~min}$. at $4{ }^{\circ} \mathrm{C}$. The cells were fixed and permeabilized using Foxp3 Fixation/Permeabilization working solution (eBioscience) according to the manufacturer's instructions. Following that, the cells were stained with anti-Foxp3 mAb for $30 \mathrm{~min}$. at RT. Data were collected on a FACSverse and analyzed with the FlowJo software.

\section{Antigen-presenting ability}

To investigate the difference in the peptide-MHC-I complex formation between the BMDCs and $\gamma$-ResDCs, each DC was stimulated with or without $100 \mathrm{ng} / \mathrm{mL}$ LPS in the presence of $500 \mu \mathrm{g} / \mathrm{mL}$ OVA protein (Sigma-Aldrich). Additionally, to investigate the ability of peptide-MHC-I complex formation induced by $\gamma$-Res in LPS-treated BMDCs, cells were stimulated with DMSO (Con), LPS, or LPS and $\gamma$-Res in the presence of 500 $\mu \mathrm{g} / \mathrm{mL}$ OVA protein. OVA $_{257-264}$ peptide (SIINFEKL) acts as a positive control. Next, to investigate the difference in the peptide-MHC-II complex formation between BMDCs and $\gamma$-Res-DCs, cells were treated with or without $100 \mathrm{ng} / \mathrm{mL}$ LPS in the presence of $25 \mu \mathrm{g} / \mathrm{mL} \mathrm{E}_{44-76}$ peptide (RLEEFAKFASFEAQGALANIAVDKANLDVMKKR; underlined sequence bound to MHC-II). To investigate the ability of peptide-MHC-II complex formation induced by $\gamma$-Res in LPS-treated BMDCs, cells were stimulated with DMSO (Con), LPS, or LPS and $\gamma$-Res in the presence of $25 \mu \mathrm{g} / \mathrm{mL} \mathrm{E}_{44-76}$ peptide. The $\mathrm{E} \alpha_{52-68}$ peptide (ASFEAQGALANIAVDKA) acts as a positive control. After $24 \mathrm{~h}$ of treatment, each cell was stained with anti-CD11c, anti-H-2Kb Ab (SIINFEKL, eBioscience) or anti-I-Ab-E $\alpha_{52-68}$ Ab (Y-Ae; ASFEAQGALANIAVDKA, eBioscience) mAbs for 20 min. at RT. Data were collected on a FACSverse and analyzed with the FlowJo software. All peptides were synthesized by AbFrontier (Seoul, Korea).

\section{Immunoblotting analyses}

To analyze the difference in signaling mechanisms of the BMDCs and $\gamma$-Res-DCs, each cell was treated with $100 \mathrm{ng} / \mathrm{mL}$ LPS in a time-dependent manner. Moreover, to analyze the effect of $\gamma$-Res in response to activation signals induced by LPS-treated BMDCs, BMDCs were treated with LPS in the presence and absence of $\gamma$-Res. For the cytosolic fraction, the cells were lysed with RIPA lysis buffer (Pierce, Rockford, IL, USA). The next steps were carried out as previously described [18]. Furthermore, nuclear extracts from cells were isolated with the CelLytic NuCLEAR Extraction Kit (Sigma-Aldrich) following the manufacturer's instructions. Western blotting Abs and HRP-conjugated anti-rabbit Abs were obtained from Santa Cruz Biotechnology, Inc. (Santa Cruz, CA, USA) and Sigma-Aldrich, respectively.

\section{DSS-induced colitis and treatment with $\gamma$-Res}

C57BL/6 mice were treated for 7 days with 2.5\% DSS (molecular weight 36-50 kDa, MP Biomedical, Aurora, $\mathrm{OH}, \mathrm{USA}$ ) dissolved in sterile water as a source of drinking water. Normal mice were given water. The mice were divided into six groups ( $n=5$ mice per group): (Group 1) normal, (Group 2) DSS + vehicle (2\% DMSO and 0.5\% Tween-20 in PBS), (Group 3) DSS $+75 \mathrm{mg} / \mathrm{kg}$ of 5-aminosalicylic acid (ASA, positive control), (Group 4) DSS $+2 \mathrm{mg} / \mathrm{kg}$ of $\gamma$-Res, and (Group 5) DSS $+5 \mathrm{mg} / \mathrm{kg}$ of $\gamma$-Res. Both ASA and $\gamma$-Res were dissolved in the vehicle solution, and this was given by oral gavage, once per day. For the survival study, five mice each in only the DSS (Group 2) and DSS $+\gamma$-Res group (Group 5) were subjected to $2.5 \%$ DSS until day 7 , and then, they were given normal distilled water and monitored for survival up to day 35 .

\section{Colitis evaluation after treatment with $\gamma$-Res}

The body weights of the mice were monitored every day. The disease activity index (DAI) was calculated by the method described by Park et al. [19]. Briefly, diarrhea (0, none; 2, loose stool; 4, watery diarrhea) and hematochezia (0, none; 2 , slight bleeding; 4 , gross bleeding) were observed during colitis induction for 7 days. At day 8 following the induction of colitis, the mice were sacrificed, and their entire colons were collected and cleaned of fat. For histological analysis, some of the colon was fixed in $10 \%$ buffered formalin and embedded in paraffin, and then, the sections were stained with hematoxylin and eosin (H\&E). To analyze the functions of $\mathrm{T}$ cells sorted from the DSS-induced mice, isolated T cells were stimulated with anti-CD3/ CD28 mAbs $(0.5 \mu \mathrm{g} / \mathrm{mL}$ each) in the presence of GolgiPlug for $9 \mathrm{~h}$, and then, the cytokine secretion ability for the activated $\mathrm{T}$ cells was analyzed using flow cytometry. 


\section{Cellular Physiology Cell Physiol Biochem 2019;52:1117-1138 \\ \begin{tabular}{ll|l} 
and Biochemistry & $\begin{array}{l}\text { DOl: 10.33594/000000076 } \\
\text { Published online: } 17 \text { April } 2019\end{array}$ & $\begin{array}{l}\text { O } 2019 \text { The Author(s). Published by } \\
\text { Cell Physiol Biochem Press GmbH\&Co. KG }\end{array}$ \\
\cline { 2 - 3 }
\end{tabular} \\ Kim et al.: Gamma-Irradiated Resveratrol Induces Tolerance and Anti-Inflammatory \\ Activity in Dendritic Cells}

\section{Statistics}

Data were determined with Tukey's multiple comparison test or unpaired t-tests using the GraphPad Prism program (San Diego, CA, USA). The data in our graphs are expressed as the means. ${ }^{*} p<0.05,{ }^{* *} p<0.01$ and ${ }^{* * *} p<0.001$ were considered statistically significant.

\section{Results}

Gamma irradiation induces structural modification of the intact-Res

Radiation technology may be used for structural modification of various small molecules $[15,16,20]$. Thus, in this study, which was conducted to analyze the effect of the structural changes of intact-Res induced by gamma irradiation, intact-Res was irradiated with a $50 \mathrm{kGy}$ dose, and the structure and molecular weight of the $\gamma$-Res using HPLC, ESI-MS, ${ }^{1} \mathrm{H}-\mathrm{NMR}$, and ${ }^{13} \mathrm{C}$-NMR were analyzed. The retention times of the intact-Res $\left(t_{\mathrm{R}}=18.1 \mathrm{~min}\right.$. $)$ and $\gamma$-Res $\left(t_{\mathrm{R}}\right.$ $=8.7$ min.) were measured (Fig. 1A) with the HPLC chromatograms. This result may predict the induced structural modification of the intact-Res by gamma irradiation; thus, a new radiolytic peak ( $\gamma$-Res) was fractionated (Fig. 1B), and the chemical structure of the $\gamma$-Res using ESI-MS (Fig. 1C), ${ }^{1} \mathrm{H}-\mathrm{NMR}$ (Supplementary Fig. S1A - for all supplemental material see www.cellphysiolbiochem.com) and ${ }^{13} \mathrm{C}-\mathrm{NMR}$ (Supplementary Fig. S1B) was analyzed. The $\gamma$-Res was identified as follows: Amorphous white powder; ${ }^{1} \mathrm{H}$ NMR (500 MHz, DMSO-D6

A

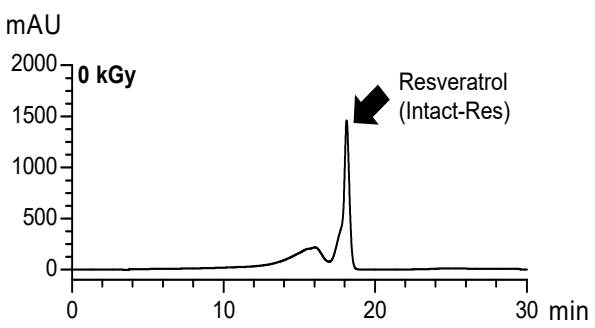

$\mathrm{mAU}$

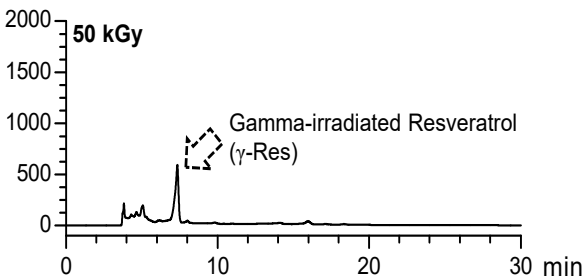

B

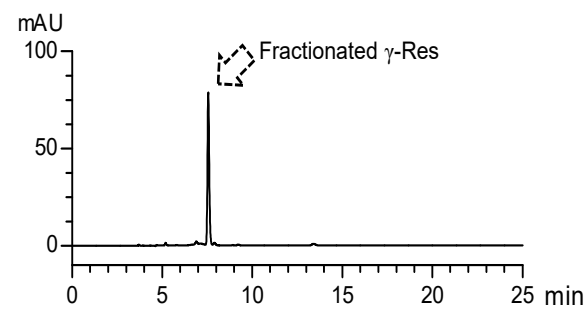

C

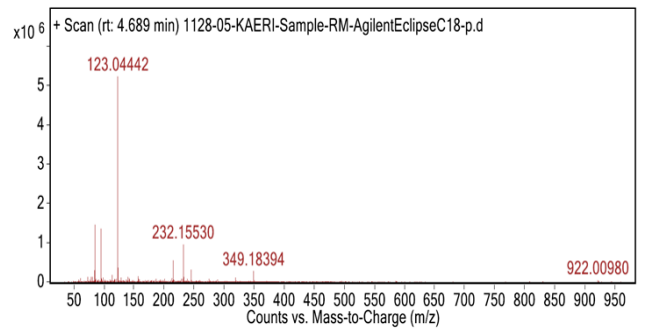

D

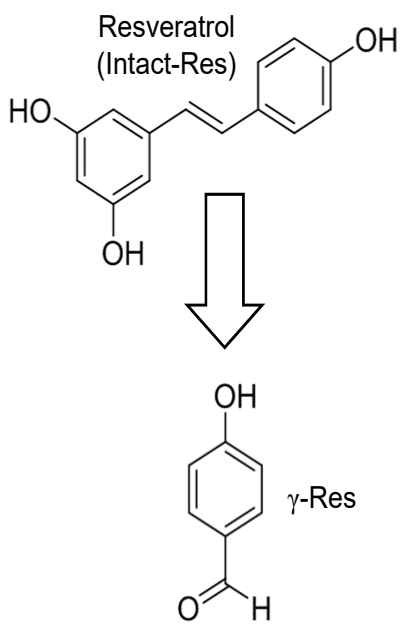

Fig. 1. Structural modification of resveratrol using gamma irradiation. A) The HPLC chromatogram of intact resveratrol (intact-Res) and gamma-irradiated resveratrol ( $\gamma$-Res) with a dose of $50 \mathrm{kGy}$ in methanol. B) The chromatogram of the fractionated $\gamma$-Res. C) The mass spectrum of $\gamma$-Res. D) Chemical structure of $\gamma$-Res. 


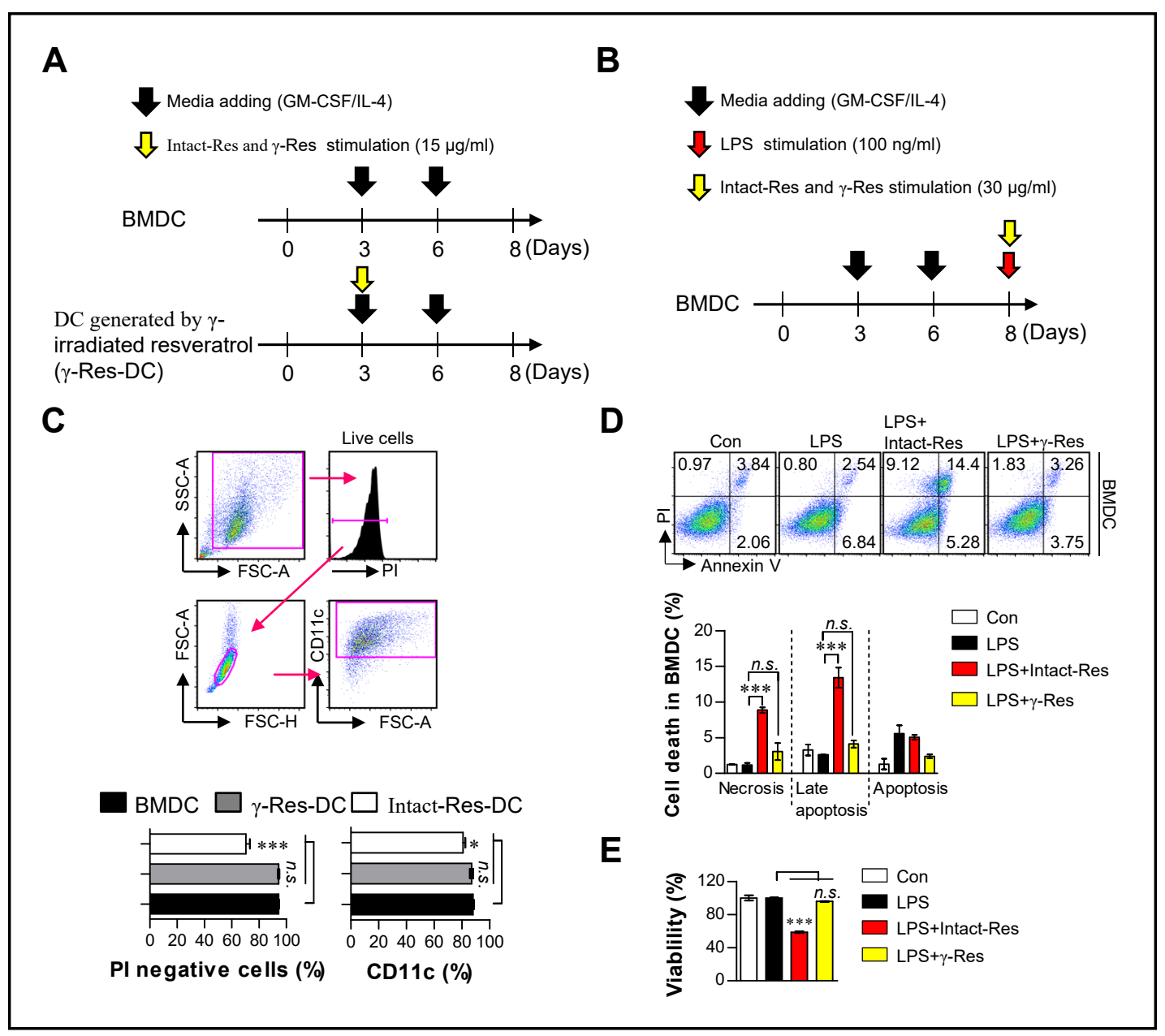

Fig. 2. Cell viability by $\gamma$-Res treatment during DC differentiation and in LPS-activated BMDCs. Schematic diagram for the phenotype and functional analysis of DCs ( $\gamma$-Res-DCs) generated by $\gamma$-Res treatment (15 $\mu \mathrm{g} /$ $\mathrm{mL}$ ) during DC differentiation (A) and the evaluation of the anti-inflammatory activity by the $\gamma$-Res treatment in LPS-activated BMDCs (B). Details regarding this experiment are provided in the Methods section (C). After DC differentiation for 8 days, BMDCs, intact-DCs and $\gamma$-Res-DCs were stained with anti-CD11c and PI Abs, and then, the cells were analyzed by flow cytometry. BMDCs were treated with LPS (100 ng/mL), LPS with intact-Res $(30 \mu \mathrm{g} / \mathrm{mL})$, or LPS with $\gamma$-Res $(30 \mu \mathrm{g} / \mathrm{mL})$ for $24 \mathrm{~h}$, and cell toxicity was determined using an Annexin V/PI staining (D) and EZ-Cytox cell viability kit (E). The results are representative of three independent experiments. All bar graphs show the means \pm SD of four samples per group. Con: untreated BMDCs, LPS-treated BMDCs (LPS), LPS+Intact-Res: BMDC treated with LPS and intact resveratrol, LPS $+\gamma-$ Res: BMDC treated with LPS and $\gamma$-Res. ${ }^{* *}$ p $<0.001$. n.s., no significant effect.

TMS), 39.737 (s, 1H, CHO), 7.719 (d, 2H, J = 8.4 Hz, 2, 6-H), 6.891 (m, 2H, 3, 5-H), and 5.951 (s, $1 \mathrm{H}, \mathrm{OH}) ;{ }^{13} \mathrm{C}$ NMR (125 MHz, DMSO-D6, ppm), $3191.434,164.307,132.640$, and 116.473, and MS (ESI, $\mathrm{m} / \mathrm{z}), 123.0444\left[\mathrm{M}+\mathrm{H}^{+}\right]$. The identified structure of the $\gamma$-Res is proposed in Fig. 1D.

$\gamma$-Res-treated cells during DC differentiation and DCs co-treated with LPS and $\gamma$-Res are not cytotoxic

Prior to conducting the study on the functional change of the DCs ( $\gamma$-Res-DCs) generated by $\gamma$-Res stimulation during DC differentiation (Fig. 2A,C) and the potential anti-inflammatory activity by $\gamma$-Res in LPS-treated BMDCs (Fig. 2B, D, E), we first identified the frequency of the $\mathrm{CD} 11 \mathrm{c}^{+}$(CD11c is a DC marker) cells and live cells (PI negative cells) following intact-Res $(15$ and $50 \mu \mathrm{g} / \mathrm{mL}$ ) and $\gamma$-Res (15 and $50 \mu \mathrm{g} / \mathrm{mL}$ ) stimulation on day 3 of DC differentiation 


\section{Cellular Physiology Cell Physiol Biochem 2019;52:1117-1138 \\ \begin{tabular}{ll|l} 
and Biochemistry $\begin{array}{l}\text { DOl: 10.33594/000000076 } \\
\text { Published online: } 17 \text { April 2019 }\end{array}$ & $\begin{array}{l}\text { O } 2019 \text { The Author(s). Published by } \\
\text { Cell Physiol Biochem Press GmbH\&Co. KG }\end{array}$ \\
\cline { 2 - 3 }
\end{tabular} \\ Kim et al.: Gamma-Irradiated Resveratrol Induces Tolerance and Anti-Inflammatory \\ Activity in Dendritic Cells}

in the presence of GM-CSF and IL-4 (Fig. 2C). As a result, on day 8 of DC differentiation, the $\gamma$-Res-DCs showed no cytotoxic effect when used at a concentration below $50 \mu \mathrm{g} / \mathrm{mL}$, and there was no change in the frequency of CD11 $\mathrm{c}^{+}$cells in the $\gamma$-Res-stimulated cells compared with the non-treated BMDCs; however, $\gamma$-Res at a concentration of $50 \mu \mathrm{g} / \mathrm{mL}$ showed a lower number of CD11 $\mathrm{c}^{+}$cells in the $\gamma$-Res-DCs compared with the normal BMDCs (data not shown). Moreover, the intact-Res treatment during DC differentiation showed an increased cell death and decreased CD11 $\mathrm{c}^{+}$population when used at a concentration below 15 (Fig. 2C) and 50 $\mu \mathrm{g} / \mathrm{mL}$ (data not shown). Based on these results, we found that the proper concentration of $\gamma$-Res was $15 \mu \mathrm{g} / \mathrm{mL}$ which had non-toxicity and no decrease in the CD11 $\mathrm{c}^{+}$numbers. Next, before identifying the anti-inflammatory activity of $\gamma$-Res in LPS-treated BMDCs, we confirmed the cytotoxic effect of $\gamma$-Res at a concentration of $30 \mu \mathrm{g} / \mathrm{mL}$ in LPS-treated BMDCs compared to the intact-Res and showed that the $\gamma$-Res-treated BMDCs in the presence of LPS for $24 \mathrm{~h}$ had no cytotoxic effect, whereas the BMDCs co-treated with LPS and intactRes lead to increased percentages of necrosis and late apoptosis cells and a decreased cell viability (Fig. 2D, E). These results indicate that $\gamma$-Res induces a stable DC differentiation and non-toxic effect in LPS-treated DCs when used at concentrations below 15 or $30 \mu \mathrm{g} / \mathrm{mL}$, respectively.

$\gamma$-Res induces the generation of IL-10-producing DCs and an anti-inflammatory action in LPS-treated BMDCs

Resveratrol has been reported as having anti-inflammatory activities, but high doses $(\geq$ $10 \mu \mathrm{g} / \mathrm{mL}$ ) of Res induce cell death in normal cells [13]. To confirm the anti-inflammatory activity of $\gamma$-Res at a non-toxic dose, we analyzed the decreased ability of cytokines secreted by LPS-treated BMDCs after treatment with $30 \mu \mathrm{g} / \mathrm{mL} \gamma$-Res or intact-Res for $24 \mathrm{~h}$. As shown in Fig. 3A, cytokines, including TNF- $\alpha$, IL-12p70 and IL-10, secreted by the LPS-treated BMDCs were reduced significantly by $\gamma$-Res or intact-Res; interestingly, $\gamma$-Res and intact-Res did not show any difference. Based on the above results, we identified the functional change (Fig. 3B) of the $\gamma$-Res-DCs and the anti-inflammatory activity (Fig. 3C) in LPS-treated BMDCs. To investigate the different ability for cytokine secretion between $\gamma$-Res-DCs and BMDCs, we analyzed the pro- (TNF- $\alpha$ and IL-12p70) and anti-inflammatory cytokine (IL-10) production of $\gamma$-Res-DCs and BMDCs in the presence and absence of LPS for $24 \mathrm{~h}$. The LPS-treated $\gamma$-ResDCs showed a lower production of TNF- $\alpha$ and IL-12p70 than that of the LPS-treated BMDCs, while IL-10 production was highly induced (Fig. 3B). Additionally, in the study on the antiinflammatory activity of $\gamma$-Res, the LPS-treated DCs that were treated with $\gamma$-Res $(1,15$, and $30 \mu \mathrm{g} / \mathrm{mL}$ ) for $24 \mathrm{~h}$ showed a significant reduction in LPS-induced TNF- $\alpha$, IL-12p70 and IL10 production compared with the LPS-treated DCs (Fig. 3C). These results were confirmed by intracellular staining, which showed that the $\gamma$-Res-DCs in the presence and absence of LPS for $12 \mathrm{~h}$ showed an increased intracellular IL-10 expression but a low expression of intracellular TNF- $\alpha$ and IL-12p70 compared with the BMDCs and LPS-treated BMDCs (Fig. $4 \mathrm{~A}, \mathrm{~B}$ ). Furthermore, the LPS $/ \gamma$-Res-treated BMDCs significantly decreased the LPS-induced TNF- $\alpha$, IL-12p70 and IL-10 production compared with the LPS-treated DCs (Fig. 4C). These results indicate that $\gamma$-Res treatment during DC differentiation may trigger the generation of IL-10-producing DCs, and $\gamma$-Res treatment in LPS-treated BMDCs may lead to an antiinflammatory activity.

Expression of surface molecules on DCs generated by $\gamma$-Res and LPS $/ \gamma$-Res-treated BMDCs The IL-10 production level is higher in tolerogenic DCs compared with immunogenic DCs [21]. Thus, we hypothesized that IL-10-producing DCs generated by $\gamma$-Res induce tolerogenic phenotypes. To validate this hypothesis, we analyzed the expression of surface molecules (CD80, CD86, MHC-I and MHC-II) on $\gamma$-Res-DCs and BMDCs after LPS treatment for $24 \mathrm{~h}$. The $\gamma$-Res-DCs in the presence and absence of LPS treatment showed a low expression of surface molecules compared with the non- or LPS-treated BMDCs (Fig. 5A, B, C). We next 


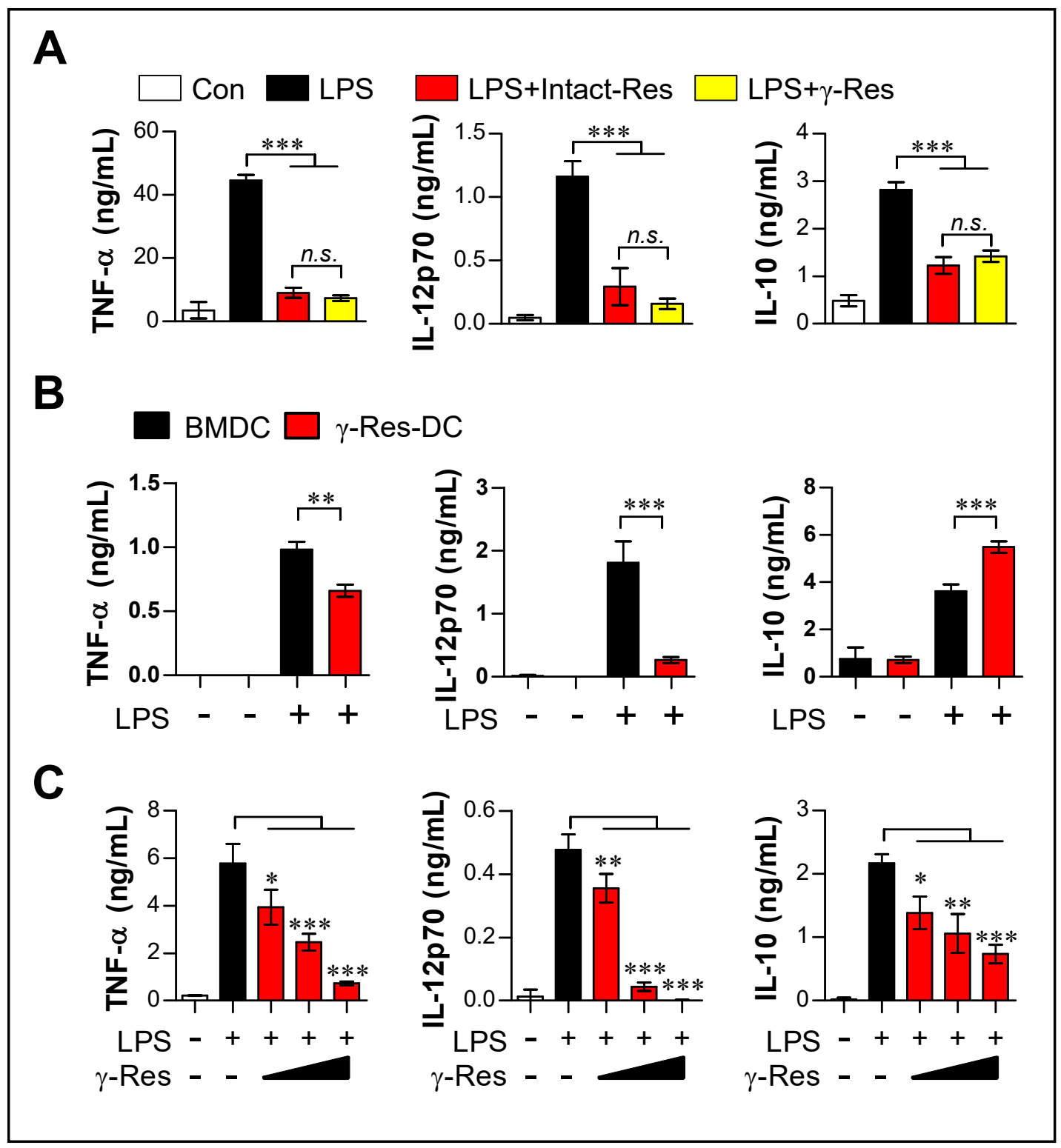

Fig. 3. Extracellular cytokine production in $\gamma$-Res-treated BMDCs and DCs generated by $\gamma$-Res in the presence of LPS. A) BMDCs were treated with intact resveratrol $(30 \mu \mathrm{g} / \mathrm{mL})$ or $\gamma$-Res $(30 \mu \mathrm{g} / \mathrm{mL})$ with LPS for $24 \mathrm{~h}$, and then, the TNF- $\alpha$, IL-12p70 and IL-10 levels in the culture medium were analyzed by ELISA. B) BMDCs and $\gamma$-Res-DCs were treated with LPS $(100 \mathrm{ng} / \mathrm{mL})$ for $24 \mathrm{~h}$, and then, the cytokine levels in the culture medium were analyzed by ELISA. C) LPS-treated BMDCs were treated with $\gamma$-Res at various doses (1, 15 , and $30 \mu \mathrm{g} / \mathrm{mL}$ ), and then, the cytokine levels in the culture medium were analyzed by ELISA. The results are representative of three independent experiments. All data were expressed as the means \pm SD ( $n=4$ samples per group). Con: untreated BMDCs, LPS+Intact-Res: BMDC treated with LPS and intact resveratrol, LPS $+\gamma$-Res: BMDC treated with LPS and $\gamma$-Res. ${ }^{*} p<0.05,{ }^{* *} \mathrm{p}<0.01$, or ${ }^{* * *} \mathrm{p}<0.001$. n.s., no significant effect.

investigated whether treatment with $\gamma$-Res resulted in a phenotypic alteration in the BMDCs upon stimulation with LPS. Thus, LPS-treated BMDCs were treated with $\gamma$-Res for $24 \mathrm{~h}$, and then, the cell surface molecules were analyzed on the BMDCs. As shown in Fig. 5D and E, CD80, CD86, MHC-I and MHC-II in the LPS $/ \gamma$-Res-treated BMDCs expressed significantly lower levels of cell surface molecules than that of the LPS-treated DCs. 


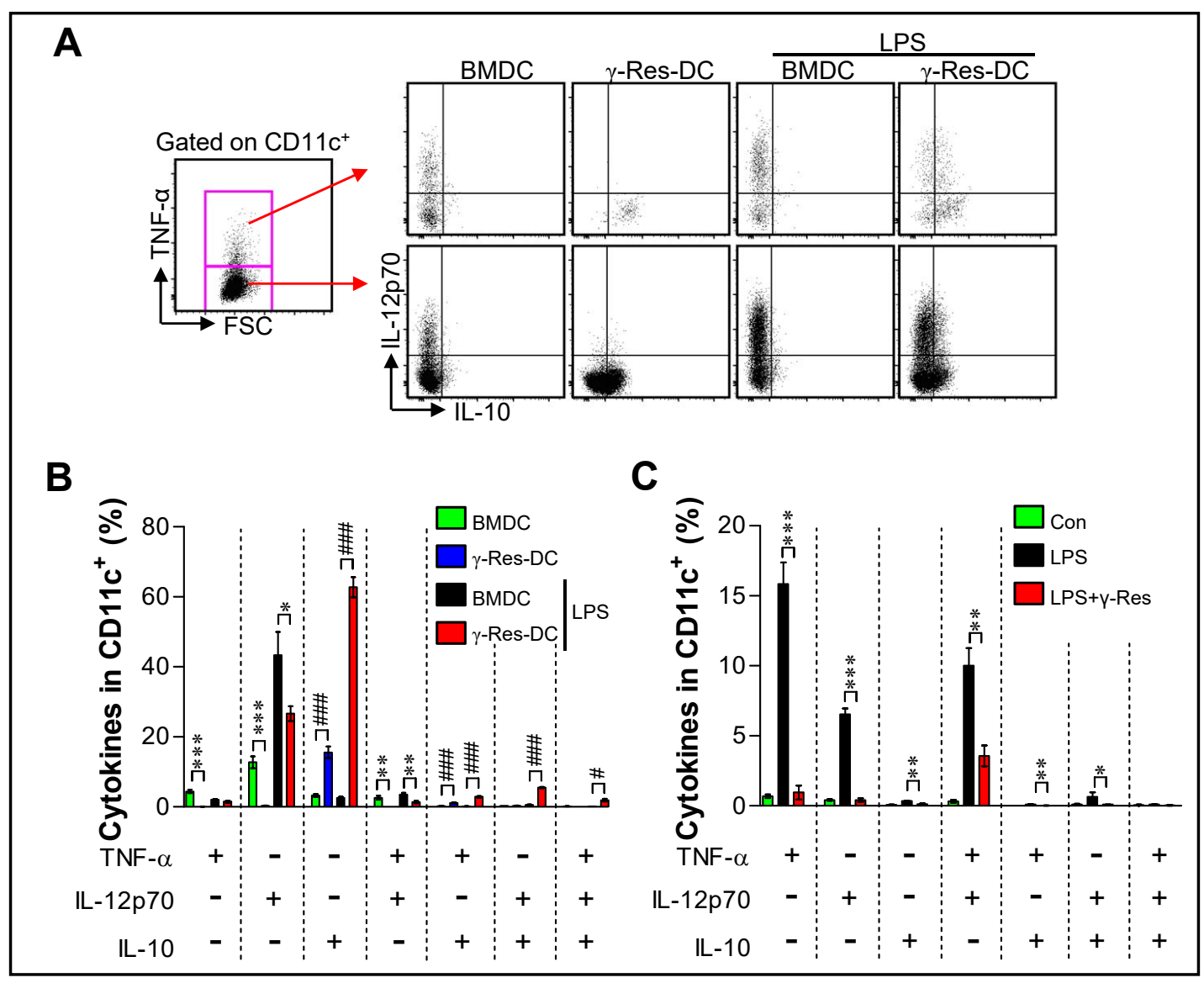

Fig. 4. Intracellular cytokine production in $\gamma$-Res-treated BMDCs and DCs generated by $\gamma$-Res in the presence of LPS. A) The BMDCs and $\gamma$-Res-DCs were incubated with LPS (100 ng/mL) in the presence of GolgiPlug for $12 \mathrm{~h}$, and stained with anti-TNF- $\alpha$, anti-IL-12p70, anti-IL-10, and anti-CD11c Abs. Intracellular levels of TNF- $\alpha$, IL-12p70 and IL-10 in CD11 ${ }^{+}$cells were analyzed by flow cytometry. B) The bar graph shows the mean \pm SD ( $n=3$ samples per group) of the percentage of intracellular cytokine levels in BMDCs and $\gamma$-Res-DCs. C) BMDCs were treated with LPS $(100 \mathrm{ng} / \mathrm{mL})$ or LPS with $\gamma$-Res $(30 \mu \mathrm{g} / \mathrm{mL})$ in the presence of GolgiPlug for $12 \mathrm{~h}$. Intracellular levels of TNF- $\alpha$, IL-12p70 and IL-10 were analyzed by flow cytometry. All results are representative of three independent experiments. All bar graphs show the means \pm SD of three samples per group. ${ }^{*} \mathrm{p}<0.05,{ }^{* *} \mathrm{p}<0.01$, or ${ }^{* * *} \mathrm{p}<0.001$.

Antigen-uptake ability of DCs generated by $\gamma$-Res and LPS $/ \gamma$-Res-treated BMDCs

Functionally, immature, tolerogenic or IL-10-producing DCs have a higher endocytic ability than that of mature DCs [22]. To evaluate the endocytic ability of the $\gamma$-Res-DCs and LPS $/ \gamma$-Res-treated BMDCs, cells were stimulated by LPS and then assessed for their endocytic ability using FITC-conjugated dextran (Fig. 6). A significantly higher percentage of dextranpositive DCs were observed among the non- and LPS-treated $\gamma$-Res-DCs compared with the non- and LPS-treated BMDCs, respectively (Fig. 6A). Moreover, the LPS $/ \gamma$-Res-treated BMDCs showed a higher percentage of dextran-positive cells than that of the LPS-treated BMDCs (Fig. 6B).

\section{Antigen-presenting ability of DCs generated by $\gamma$-Res and LPS $/ \gamma$-Res-treated BMDCs}

We next analyzed the antigen-presenting ability of the $\gamma$-Res-DCs (Fig. 7A, B, C) and LPS $/ \gamma$-Res-treated BMDCs (Fig. 7D) with anti-25-D1.16 mAb, which recognizes the $0 \mathrm{VA}_{257}$ ${ }_{264}$ peptide bound to the $\mathrm{H}-2 \mathrm{~Kb}$ of MHC-I, and the anti-Y-Ae mAb, which directly reacts with the $E \alpha_{52}{ }_{68}$ peptide MHC-II. For the experiment on the antigen-presenting ability of DCs generated by $\gamma$-Res, the $\gamma$-Res-DCs and BMDCs were treated with the $\mathrm{E}_{44}{ }^{-}{ }_{76}$ peptide or OVA 
A
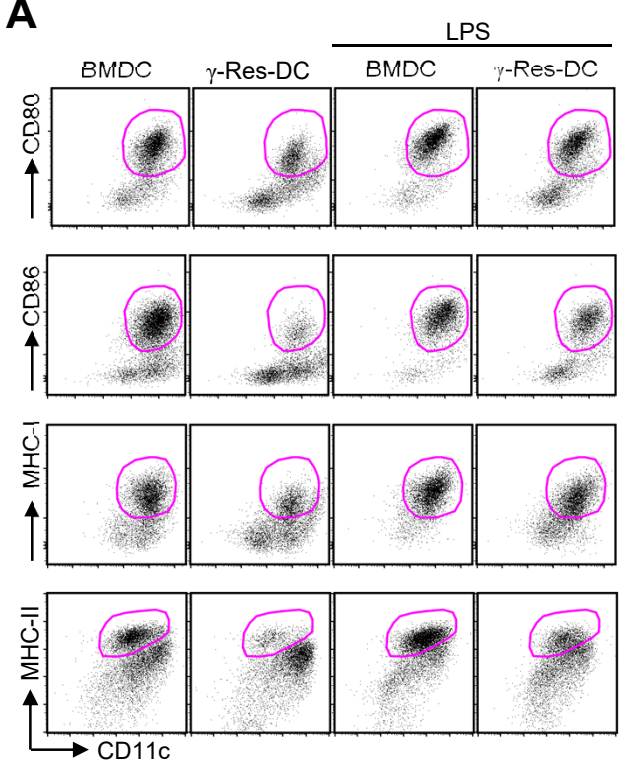

D
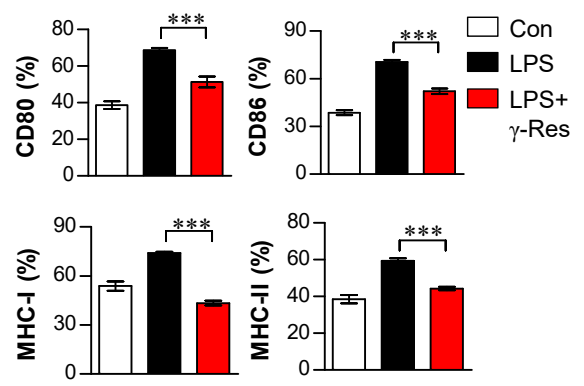

B

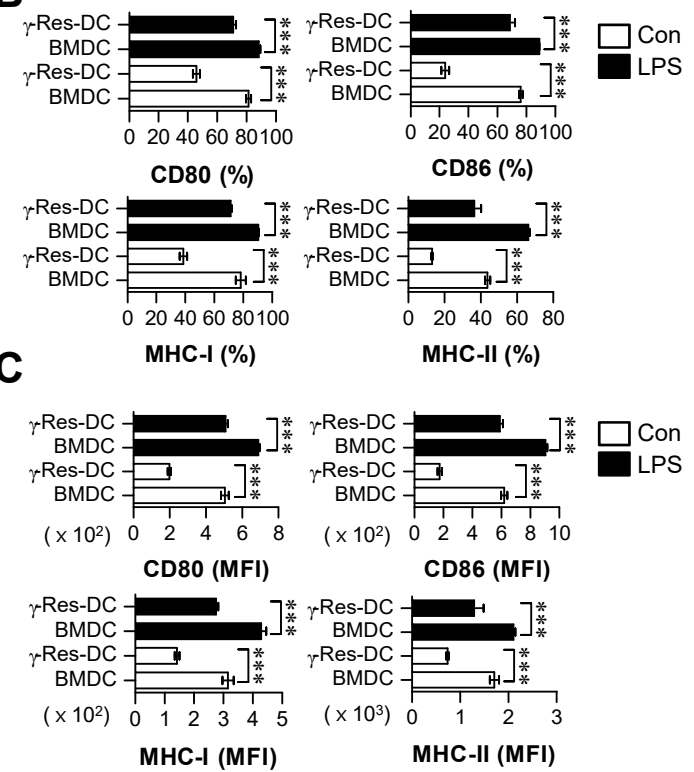

E
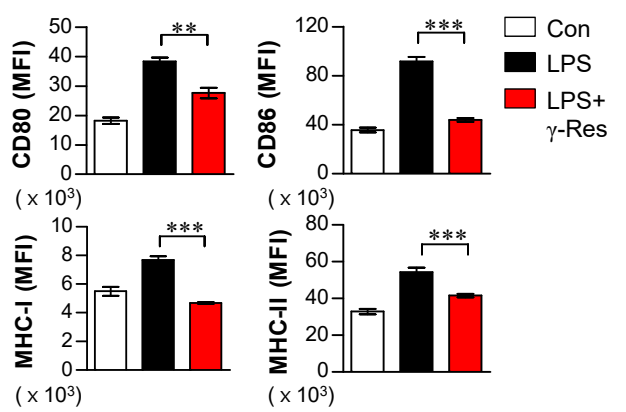

$\left(\times 10^{3}\right)$

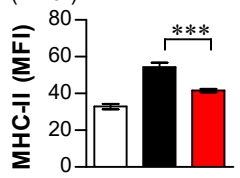

$\left(\times 10^{3}\right)$

Fig. 5. Expression of co-stimulatory molecules and MHC class molecules in $\gamma$-Res-treated BMDCs and DCs generated by $\gamma$-Res in the presence of LPS. A-C) The BMDCs and $\gamma$-Res-DCs were treated with LPS (100 ng/ $\mathrm{mL}$ ). After incubation for $24 \mathrm{~h}$, the cells were stained with anti-CD80, anti-CD86, anti-MHC-I, anti-MHC-II, and anti-CD11c Abs and analyzed by flow cytometry. The bar graphs show the means \pm SD $(n=3$ samples per group) of the percentage (B) or median fluorescence intensity (MFI) (C) of each surface molecule expressed by CD $11 \mathrm{c}^{+}$cells. D, E) The BMDCs were treated with LPS (100 ng/mL) or LPS with $\gamma$-Res (30 $\left.\mu \mathrm{g} / \mathrm{mL}\right)$ and analyzed for expression of surface markers CD80, CD86, MHC-I, MHC-II, and CD11c by flow cytometry. The bar graphs show the mean \pm SD ( $n=3$ samples per group) of the percentage (D) or MFI (E) of each surface molecule expressed by CD $11 \mathrm{c}^{+}$cells. All results are representative of three independent experiments. All bar graphs show the means \pm SD of three samples per group. ${ }^{* *} \mathrm{p}<0.01$, or ${ }^{* * *} \mathrm{p}<0.001$.

protein in the presence and absence of LPS for $24 \mathrm{~h}$, and then, the cells were stained with fluorescein-conjugated anti-Y-Ae mAb or anti-25-D1.16, respectively. Additionally, the LPS/ $\gamma$-Res-treated BMDCs and BMDCs (non-treated BMDCs) were observed for their antigenpresenting ability according to the same method used in Fig. 7A and B (Fig. 7D). $\mathrm{E} \alpha_{52^{-}{ }_{68}}$ and $\mathrm{OVA}_{257-264}$ were used as the positive control. A significantly lower percentage of $\mathrm{E} \alpha_{52}{ }^{-}{ }_{68} / \mathrm{MHC}-\mathrm{I}$ complexes (25-D1.16-positive cells) and $\mathrm{OVA}_{257-264} / \mathrm{MHC}$-II complexes (Y-Ae-positive cells) were present in the non-treated $\gamma$-Res-DCs or LPS-treated $\gamma$-Res-DCs compared with the non-treated BMDCs or LPS-treated BMDCs, respectively (Fig. 7A, B, C). We also found that the LPS $/ \gamma$-Res-treated BMDCs had a reduced percentage of $\mathrm{E}_{52}{ }^{-}{ }_{68} / \mathrm{MHC}$-I complexes and $\mathrm{OVA}_{257-264} /$ MHC-II complexes compared with the LPS-treated BMDCs (Fig. 7D). 


\section{A}
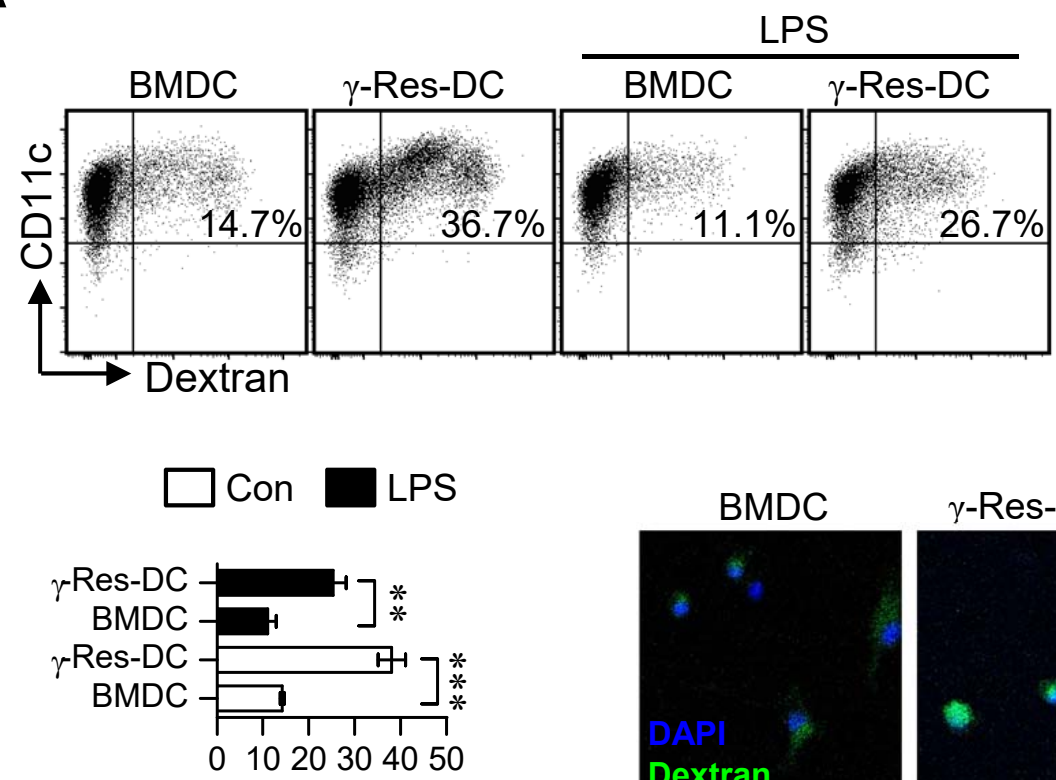

\section{CD11c $+{ }^{+}$extran ${ }^{+}(\%)$}
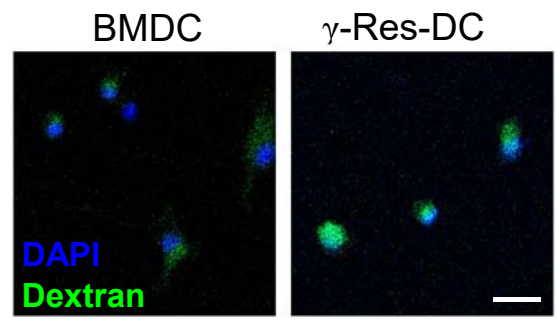

B
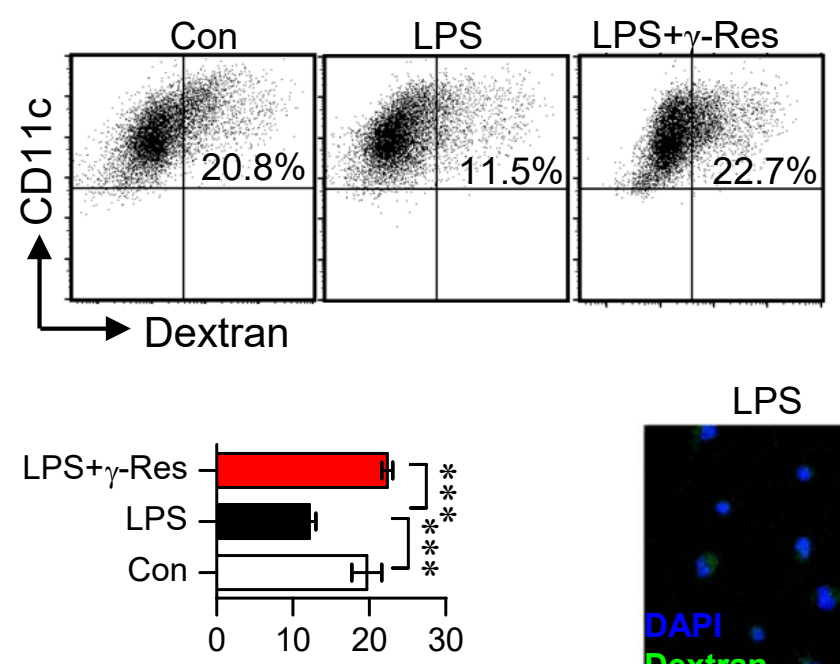

\section{Dextran (\%)}
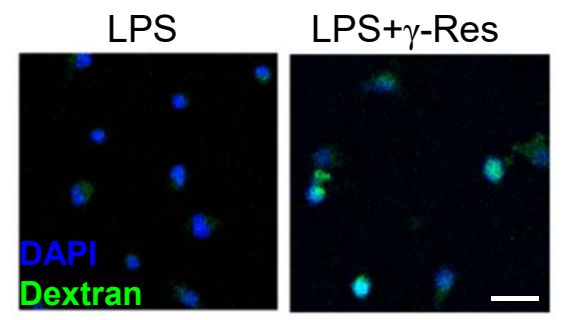

Fig. 6. Endocytic activity in $\gamma$-Res-treated BMDCs and DCs generated by $\gamma$-Res in the presence of LPS. A) The BMDCs and $\gamma$-Res-DCs were treated with LPS $(100 \mathrm{ng} / \mathrm{mL})$ for $24 \mathrm{~h}$ at $37^{\circ} \mathrm{C}$ and then incubated with $1 \mathrm{mg} / \mathrm{mL}$ FITC-dextran for $30 \mathrm{~min}$. Cells were stained with anti-CD11c Ab, and FITC-dextran-positive CD11 $\mathrm{c}^{+}$-positive cells were analyzed by flow cytometry. The endocytic capacity of the BMDCs and $\gamma$-Res-DCs was determined by assessing the endocytosis of FITC-dextran by flow cytometry (top panel) and confocal microscopy (bottom panel). B) The BMDCs were treated with LPS (100 ng/mL) or LPS with $\gamma$-Res ( $30 \mu \mathrm{g} / \mathrm{mL})$ for $24 \mathrm{~h}$ at $37^{\circ} \mathrm{C}$ and then incubated with $1 \mathrm{mg} / \mathrm{mL}$ FITC-dextran for $30 \mathrm{~min}$. The endocytic capacity of the LPS-treated BMDCs and LPS $+\gamma$-Res-treated BMDCs was determined by assessing the endocytosis of FITC-dextran by flow cytometry (top panel) and confocal microscopy (bottom panel). All results are representative of three independent experiments. All bar graphs show the mean percentages of dextran ${ }^{+} \mathrm{CD} 11 \mathrm{c}^{+}$cells $\pm S D(n=3$ samples per group). ${ }^{* *}<<0.01$, or ${ }^{* * *} \mathrm{p}<0.001$. 
A

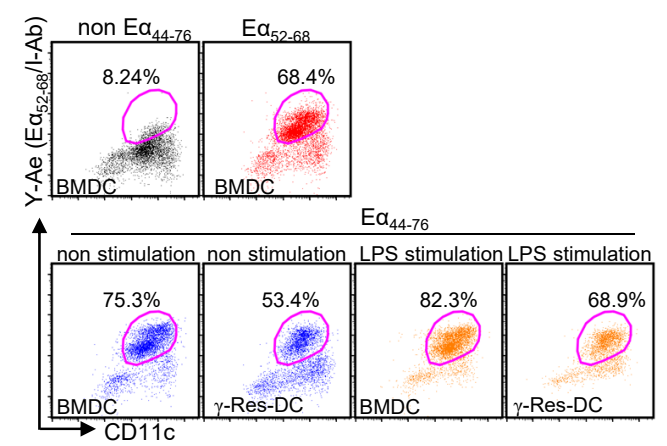

C

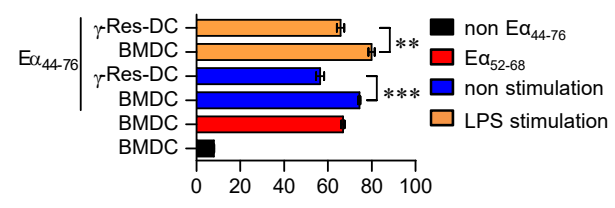

Y-Ae (\%)

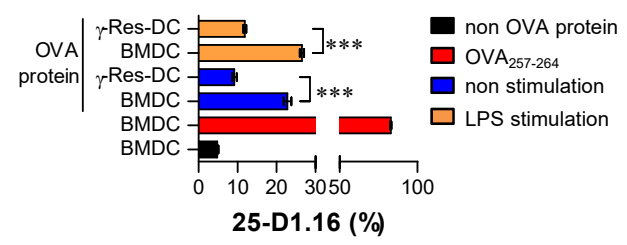

B

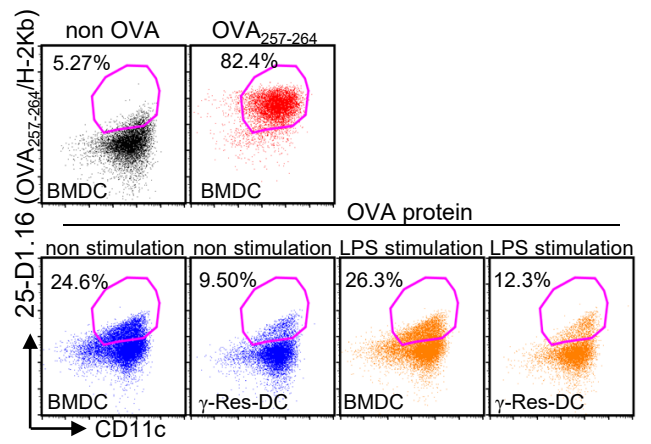

D
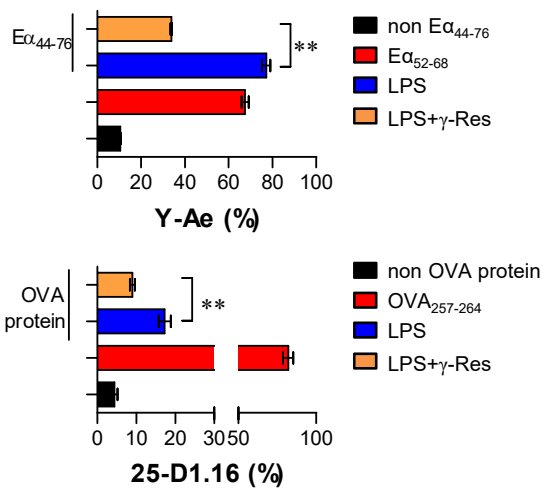

Fig. 7. Antigen-presenting ability in $\gamma$-Res-treated BMDCs and $\gamma$-Res-DCs in the presence of LPS. A-C) The BMDCs and $\gamma$-Res-DCs were treated with OVA $(500 \mu \mathrm{g} / \mathrm{mL})$ or E $\alpha$ peptide (aa 44-76; $50 \mu \mathrm{g} / \mathrm{mL})$ in the absence or presence of LPS (100 ng/mL). After incubation for $24 \mathrm{~h}$, the cells were stained with anti-CD11c, anti-25-D1.16, or anti-Y-Ae Abs for $20 \mathrm{~min}$. at room temperature. Dot plots for the expression of the $\mathrm{E} \alpha_{52-}$ ${ }_{68} / \mathrm{I}-\mathrm{Ab}$ complexes $\left(\mathrm{C}\right.$; top panel) and $\mathrm{OVA}_{257-264} / \mathrm{H}-2 \mathrm{~Kb}\left(\mathrm{C}\right.$; bottom panel) on the gated $\mathrm{CD} 11 \mathrm{c}^{+}$population are indicated. D) BMDCs (black bar), LPS-treated BMDC (blue bar) and LPS $+\gamma$-Res-treated BMDCs (orange bar) were treated with OVA $(500 \mu \mathrm{g} / \mathrm{mL}$ ) or E $\alpha$ peptide (aa 44-76; $50 \mu \mathrm{g} / \mathrm{mL}$ ) for $24 \mathrm{~h}$. Dot plots for the expression of the $\mathrm{E} \alpha_{52-68} / \mathrm{I}-\mathrm{Ab}$ complexes (top panel) and $\mathrm{OVA}_{257-264} / \mathrm{H}-2 \mathrm{~Kb}$ (bottom panel) on the gated CD11 $\mathrm{c}^{+}$population are indicated. $\mathrm{OVA}_{257-264}$ or $\mathrm{E} \alpha_{52-68}$ peptide $(1 \mu \mathrm{g} / \mathrm{mL}$ of each peptide); positive control. All results are representative of three independent experiments. All bar graphs show the means \pm SD of three samples per group. ${ }^{* *} \mathrm{p}<0.01$, or ${ }^{* * *} \mathrm{p}<0.001$.

Role of MAPKs and NF- $\kappa B$ signals in DCs generated by $\gamma$-Res and LPS $/ \gamma$-Res-treated BMDCs MAPKs and NF- $\kappa B$ signals regulate cytokine expression [23]. To examine whether these signals are involved in the increased IL-10 production of DCs generated by $\gamma$-Res and in the increased anti-inflammatory activity of LPS-treated BMDCs following treatment with $\gamma$-Res, the phosphorylated MAPKs, the nuclear translocation of NF- $\kappa B$ p65, and the phosphorylation / degradation of IкB- $\alpha$ were investigated by western blot analysis (Fig. 8). In the LPS-treated $\gamma$-Res-DCs, a rapid and prolonged ERK, JNK and p38 phosphorylation and non-activated IKB- $\alpha$ phosphorylation/degradation were observed compared to the LPS-treated BMDCs (Fig. 8A). Based on the above results, we hypothesized that IL-10 production induced by $\gamma$-Res-DCs is regulated by ERK, JNK and p38 phosphorylation. Thus, we did an experiment using the pharmacological inhibitors for ERK, JNK and p38 and then, evaluated the IL-10 production in LPS-treated $\gamma$-Res-DCs. As a result, in the presence of pharmacological inhibitors for ERK, JNK and p38, the LPS-treated $\gamma$-Res-DCs significantly abrogated the IL-10 production compared with the LPS-treated $\gamma$-Res-DCs in the absence of pharmacological inhibitors (Fig. 8B). These 


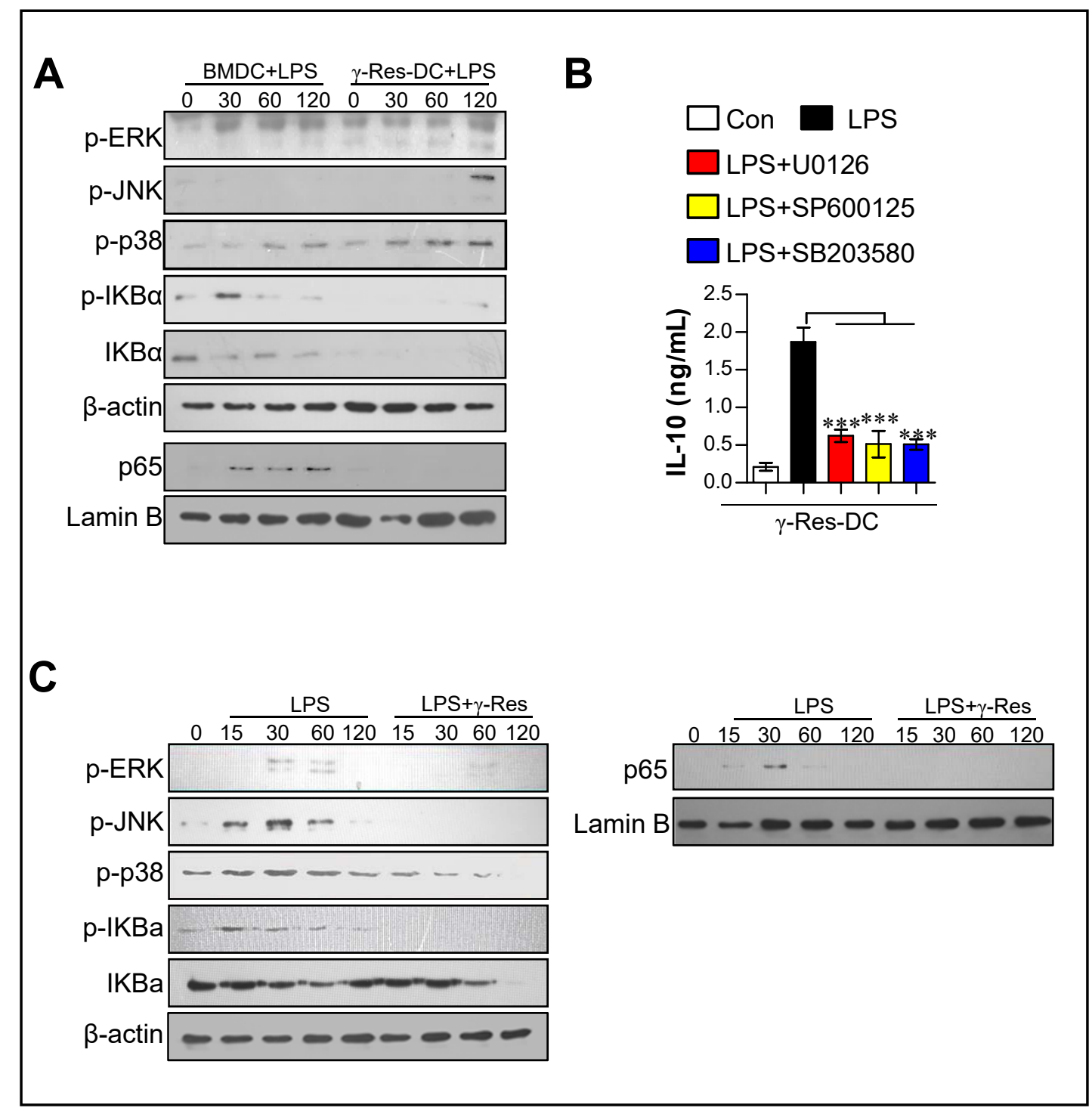

Fig. 8. Expression of MAPK and NF- $\kappa B$ signaling pathways in $\gamma$-Res-treated BMDCs and DCs generated by $\gamma$-Res in the presence of LPS. A) BMDCs and $\gamma$-Res-DCs were treated with LPS (100 ng/mL) for 0, 30, 60, and $120 \mathrm{~min}$. Cell lysates and nuclear lysates were subjected to SDS-PAGE and immunoblot analysis using each specific Abs. B) $\gamma$-Res-DCs were treated with pharmacological inhibitors of ERK (U0126), JNK (SP600125), and p38 (SB203580) for $1 \mathrm{~h}$ prior to treatment with LPS for $24 \mathrm{~h}$, and then, the IL-10 levels in the culture medium were analyzed by ELISA. The values shown represent the mean \pm SD ( $n=3$ samples per group). ${ }^{* * *} \mathrm{p}<0.001$. C) BMDCs were treated with LPS $(100 \mathrm{ng} / \mathrm{mL})$ in the absence or presence of $\gamma$-Res $(30 \mu \mathrm{g} / \mathrm{mL})$ for $0,15,30,60$, and $120 \mathrm{~min}$. Immunoblot analysis of the cell lysates and nuclear lysates was performed as described above. All results are representative of three independent experiments.

results indicate that MAPK phosphorylation is the main pathway for the IL-10 production induced by the $\gamma$-Res-DCs. Moreover, in the LPS $/ \gamma$-Res-treated BMDCs, the phosphorylated MAPKs, the nuclear translocation of NF- $\mathrm{KB}$ p65, and the phosphorylation/degradation of IкB- $\alpha$ were decreased compared to the LPS-treated BMDCs (Fig. 8C). These results also indicate that the MAPK and NF- $\mathrm{BB}$ signals are key players in the anti-inflammatory activity of the LPS-treated BMDCs following treatment with $\gamma$-Res. 


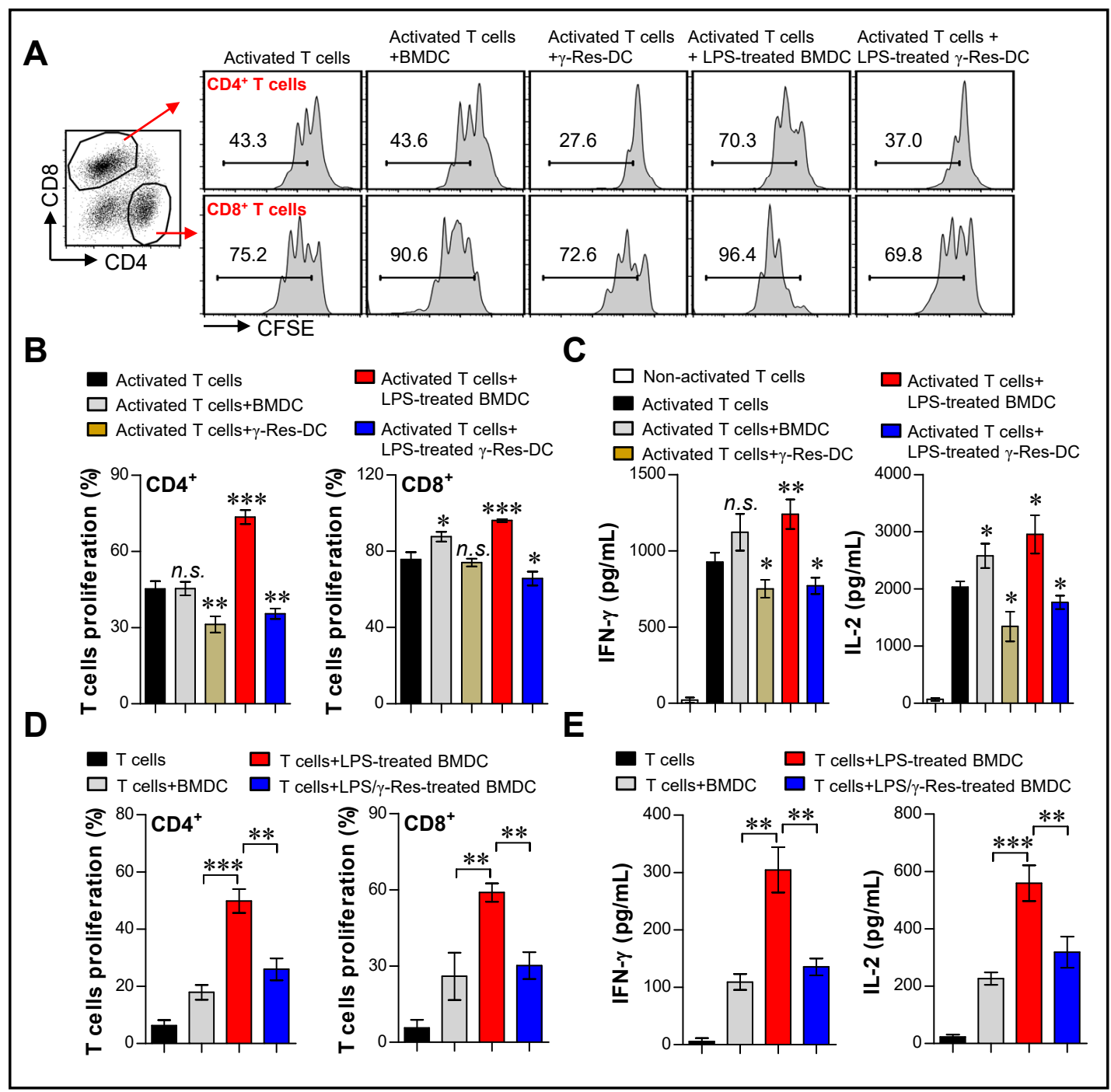

Fig. 9. Inhibitory effect of $\gamma$-Res-treated BMDCs and DCs generated by $\gamma$-Res on proliferation and activation of T cells. A-C) T cells isolated from the splenocytes of BALB/c and stained with CFSE. The stained T cells were cultured in a 48-well plate coated with anti-CD3 $(0.5 \mu \mathrm{g} / \mathrm{mL})$ and anti-CD28 Abs $(0.5 \mu \mathrm{g} / \mathrm{mL})$. BMDCs and $\gamma$-Res-DCs were stimulated with or without LPS for $1 \mathrm{~h}$ and co-cultured with the T cells. The ratio between the DCs and T cells was 0.5:1. After 3 days of co-culturing, the cells were harvested and stained with anti-CD4 and anti-CD8 Abs. B) The proliferation in activated-CD4 ${ }^{+}$and $\mathrm{CD}^{+} \mathrm{T}$ cells was analyzed using flow cytometry. D) Culture mediums were analyzed for the production of IFN- $\gamma$ and IL-2 cytokines using ELISA. D, E) BMDCs were stimulated with LPS in the absence or presence of $\gamma$-Res $(30 \mu \mathrm{g} / \mathrm{mL})$ for $24 \mathrm{~h}$ and then co-cultured with $\mathrm{T}$ cells isolated from BALB/c for 3 days. The proliferation of $\mathrm{T}$ cells and cytokines were measured as described above. All results are representative of three independent experiments. All bar graphs show the means \pm SD of three samples per group. ${ }^{*} \mathrm{p}<0.05,{ }^{* *} \mathrm{p}<0.01$, or ${ }^{* * *} \mathrm{p}<0.001$.

\section{Proliferation and activation of $T$ cells by DCs generated by $\gamma$-Res and LPS $/ \gamma$-Res-treated} BMDCs

IL-10-producing DCs have been reported to inhibit T cell activation and proliferation [24]. Thus, to precisely characterize the role of the $\gamma$-Res-DCs and T cell interaction, we performed an MLR assay using allogeneic T cells activated with CD3/CD28 antibodies (Fig. 9A, B, C). $\gamma$-Res-DCs, BMDCs, LPS-treated $\gamma$-Res-DCs and LPS-treated BMDCs were co-cultured with CFSE-labeled T cells in the presence of CD3/CD28 antibodies. Interestingly, the $\gamma$-ResDCs and LPS-treated $\gamma$-Res-DCs reduced or did not change the proliferation of both CD $4^{+}$and 


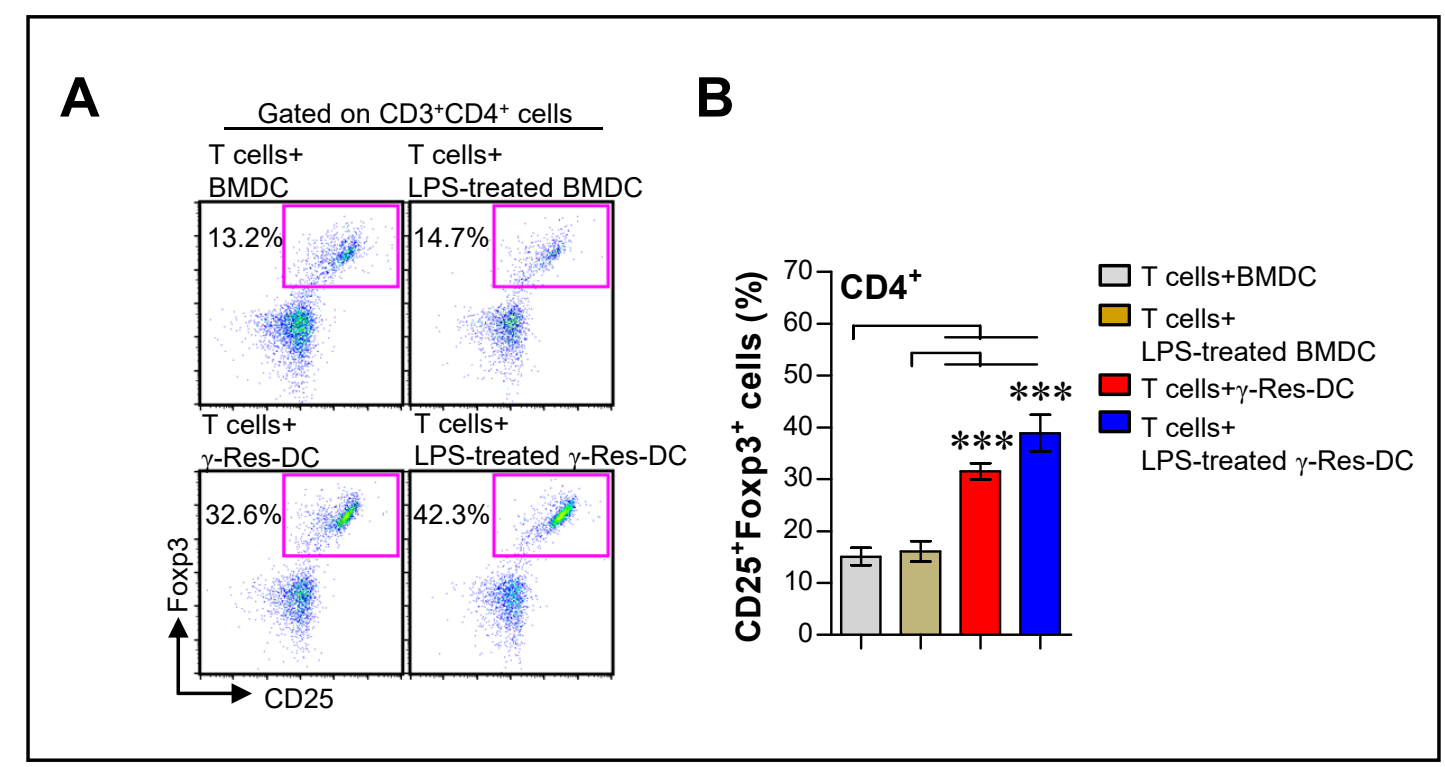

Fig. 10. Induction of regulatory $T$ cells by DCs generated by $\gamma$-Res. A, B) CD4+ T cells were isolated as described in the Methods section. BMDCs and $\gamma$-Res-DCs were co-cultured with isolated CD4+ $\mathrm{T}$ cells for 3 days and stained with anti-CD25 and anti-Foxp3Abs. The $\mathrm{CD} 25^{+} \mathrm{Foxp} 3^{+} \mathrm{CD} 4^{+} \mathrm{T}$ cells were analyzed by flow cytometry. B) The bar graph shows the percentage of CD25+Foxp $3^{+}$regulatory T cells among the CD $4^{+}$ $\mathrm{T}$ cells. All results are representative of three independent experiments. The values shown represent the mean \pm SD ( $n=3$ samples per group). ${ }^{* * *} \mathrm{p}<0.001$.

$\mathrm{CD}^{+} \mathrm{T}$ cells and the production of IFN- $\gamma$ and IL-2 compared with activated T cells, while the BMDCs and LPS-treated BMDCs, respectively, did not change or become induced (Fig. 9A, B, C). Furthermore, we evaluated the capacity of the LPS $/ \gamma$-Res-treated BMDCs to induce $\mathrm{T}$ cell proliferation and activation using allogeneic T cells. As shown in Fig. 9D and E, T cell proliferation and production of IFN- $\gamma$ and IL- 2 were not induced by the LPS $/ \gamma$-Res-treated BMDCs compared to the LPS-treated BMDCs. These finding indicate that the $\gamma$-Res-DCs and the presence of $\gamma$-Res in the LPS-treated BMDCs led to the inhibitory effect on the T cells.

\section{Induction of regulatory $T$ cells by DCs generated by $\gamma$-Res}

As a next step, we investigated whether regulatory $\mathrm{T}$ cells (Foxp $3^{+} \mathrm{CD} 25^{+} \mathrm{CD} 4^{+} \mathrm{T}$ cells) are induced during $\gamma$-Res-DC and CD4 T cell interaction because IL-10-producing DCs can induce the differentiation of naïve T cells towards regulatory T cells [21]. Thus, we evaluated the capacity of the $\gamma$-Res-DCs to induce regulatory $\mathrm{T}$ cells using the same experimental conditions described in Fig. 9B and C. As shown in Fig. 10, the $\gamma$-Res-DCs and LPS-treated $\gamma$-Res-DCs polarized naïve CD4+ $\mathrm{T}$ cells towards the regulatory T cells, while BMDCs and LPStreated BMDCs were not induced.

\section{Protective effect by the $\gamma$-Res treatment in a DSS-induced colitis model}

The above results regarding the $\gamma$-Res-generated IL-10-producing DCs and antiinflammatory activity motivated us to test the protective efficacy of $\gamma$-Res against DSSinduced colitis. For this purpose, we evaluated the body weight, disease activity index (DAI) score, colon length, colon histology, and survival rate following $\gamma$-Res treatment in $2.5 \%$ DSS-induced mice (Fig. 11A, experimental design indicated by the schematic diagram). For body weight, the $5 \mathrm{mg} / \mathrm{kg}$ dose $\gamma$-Res-treated DSS-induced groups showed a significantly increased body weight on day 5 compared with the DSS control groups, but there was no statistical difference on the other days and in the $2 \mathrm{mg} / \mathrm{kg}$ dose $\gamma$-Res-treated group (Fig. 11B). For the DAI score, the ASA-treated ( 4 days; $P<0.05,7$ days; $P<0.05$ ), $2 \mathrm{mg} / \mathrm{kg}$ (4 days; $P<0.05,7$ days; $n . s$.) and $5 \mathrm{mg} / \mathrm{kg}$ ( 4 days; $P<0.01,7$ days; $P<0.01$ ) dose $\gamma$-Res-treated DSSinduced groups had a significantly lower score than that of the DSS control groups (Fig. 11C). 


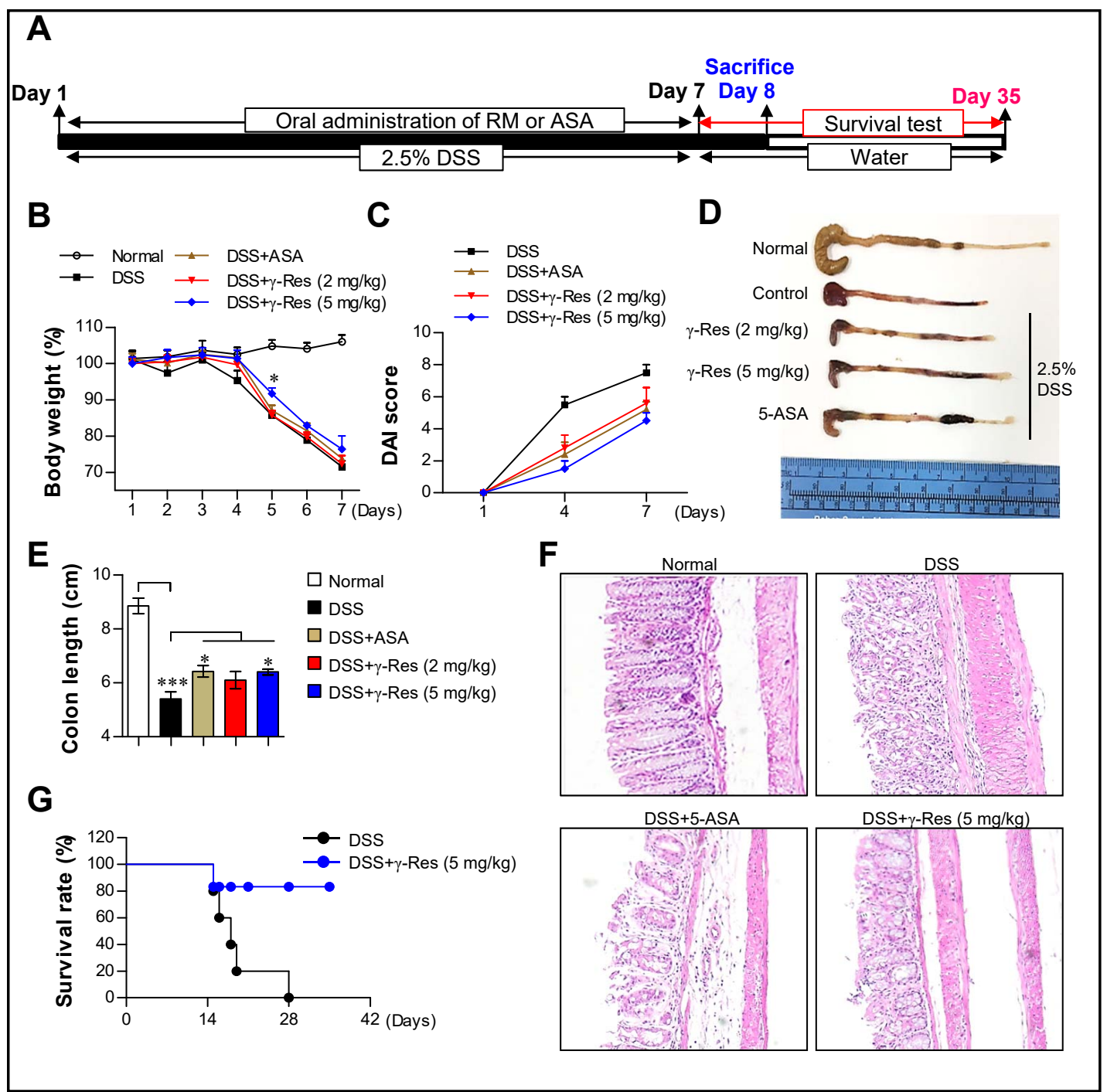

Fig. 11. Oral administration of $\gamma$-Res attenuated the clinical sign of colitis and macroscopic signs of inflammation in the colon tissue. A) Schematic diagram of the $\gamma$-Res treatment and induction of the colitis mice model via DSS. Details regarding this experiment are provided in the Methods section. B) Percentage change in body weight. C) DAI score. D) Representative photographs showing the colon tissue from each group. E) Changes in colon length. The bar graph shows the means \pm SD of 5 mice per group. ${ }^{*} \mathrm{p}<0.05$, or ${ }^{* * *} \mathrm{p}<0.001$. F) Representative histological images of the large intestine. G) Comparison of the survival rate was performed with only DSS and DSS $+\gamma$-Res $5 \mathrm{mg} / \mathrm{kg}$. Percentage survival data of $\mathrm{n}=5$ mice per group. All mice drank water containing $2.5 \%$ DSS for 7 days and then normal water.

For colon length, the $5 \mathrm{mg} / \mathrm{kg}$ dose $\gamma$-Res-treated DSS-induced groups had a significantly longer colon than that of the DSS control groups, but there was no statistical difference in the $2 \mathrm{mg} / \mathrm{kg}$ dose $\gamma$-Res-treated group (Fig. 11D, E). Additionally, the $5 \mathrm{mg} / \mathrm{kg}$ dose $\gamma$-Res-treated DSS-induced groups showed moderate to mild signs of inflammation in the colon histology when compared to the DSS control groups (Fig. 11F). Finally, the $5 \mathrm{mg} / \mathrm{kg}$ dose $\gamma$-Res-treated DSS-induced groups had a dramatically prolonged survival with severe colitis (Fig. 11G). 


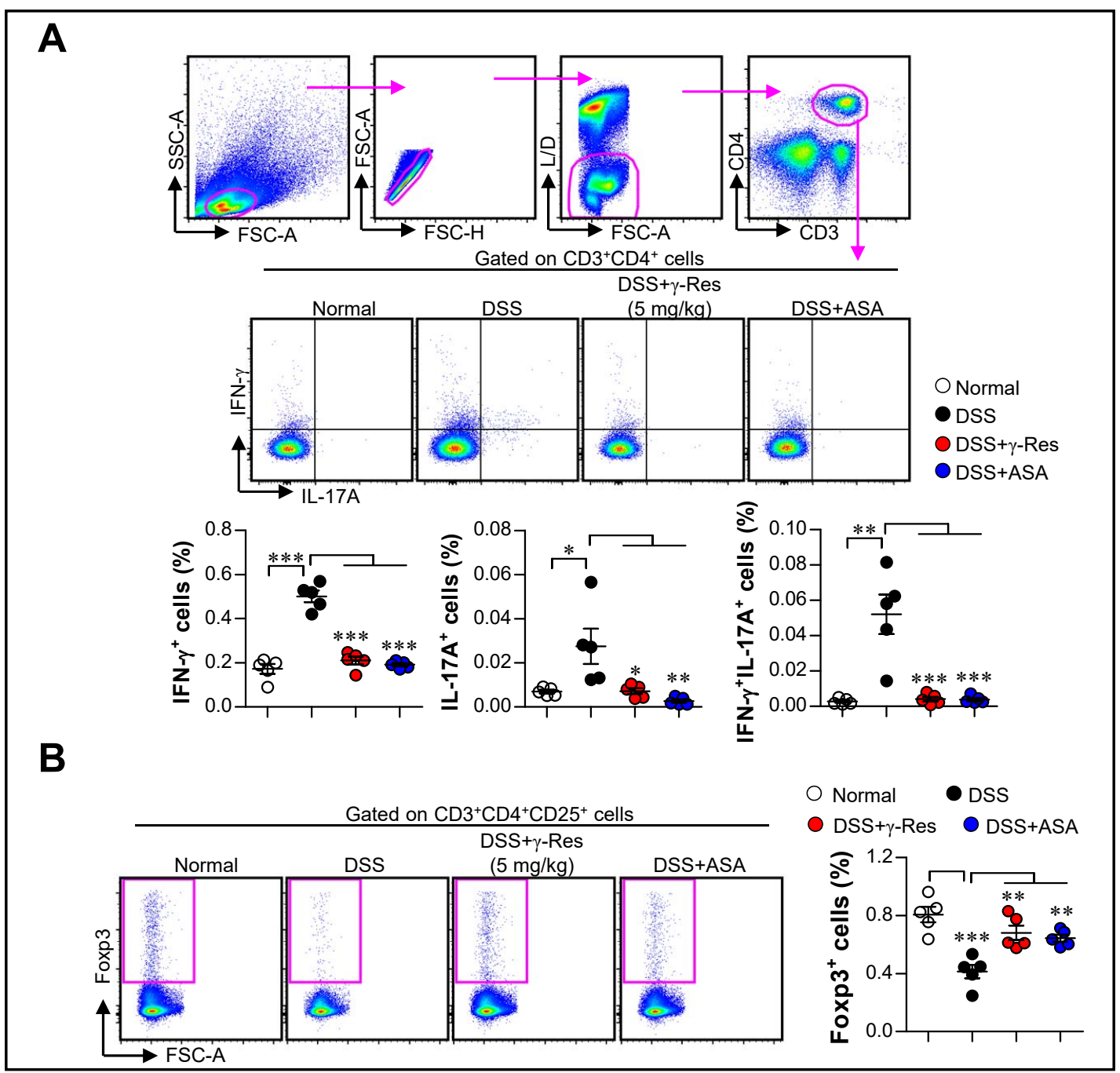

Fig. 12. Oral administration of $\gamma$-Res induced the reduction of Th1 and Th17 cells and the induction of regulatory T cells in DSS-induced colitis mice. A) Cells isolated from splenocytes of each group were stimulated with anti-CD3/CD38 Abs in the presence of GolgiPlug for $9 \mathrm{~h}$, and then, the cells were stained LIVE/DEAD (L/D), anti-CD3, anti-CD4, anti-IFN- $\gamma$, anti-IL-17A Abs and analyzed by flow cytometry. Lymphocytes were then gated based on their characteristic patterns of forward scatter and side scatter, and dead cells were excluded by gating out cells with positive staining with the LIVE/DEAD viability dye (A; gating strategy). The graphs show the percentage of each of the $\mathrm{CD}^{+} \mathrm{CD} 4^{+} \mathrm{IFN}-\gamma^{+}, \mathrm{CD}^{+} \mathrm{CD} 4^{+} \mathrm{IL}-17 \mathrm{~A}^{+}$, and $\mathrm{CD}^{+} \mathrm{CD} 4^{+} \mathrm{IFN}-\gamma^{+} \mathrm{IL}-17 \mathrm{~A}^{+} \mathrm{T}$ cells. B) Cells isolated from the splenocytes of each group were stimulated with anti-CD3/CD38 Abs for $18 \mathrm{~h}$. The graphs show the percentage of $\mathrm{CD}^{+}{ }^{+} \mathrm{CD} 4{ }^{+} \mathrm{CD} 25^{+} \mathrm{Foxp} 3^{+}$regulatory $\mathrm{T}$ cells. The values shown represent the mean $\pm \mathrm{SD}$ ( $\mathrm{n}=5$ mice of group). ${ }^{*} \mathrm{p}<0.05,{ }^{* *} \mathrm{p}<0.01$, or ${ }^{* * *} \mathrm{p}<0.001$.

Immune responses induced by the $\gamma$-Res treatment in the DSS-induced colitis model

The effective inhibition of activated CD4 $\mathrm{T}$ cell responses is an important step for generating protective immunity against IBD [25]. Therefore, we next evaluated how the $\gamma$-Res treatment affected T cell activation. After 8 days of DSS induction, splenocytes were stimulated in vitro with CD3/CD28 antibodies, and the phenotypes of the responding CD4 ${ }^{+}$ $\mathrm{T}$ cells were evaluated by intracellular cytokine staining. As shown in Fig. 12A, the $5 \mathrm{mg} /$ $\mathrm{kg}$ dose $\gamma$-Res-treated DSS-induced groups showed decreased levels of IFN- $\gamma^{+} \mathrm{CD} 4^{+}$, IL$17 \mathrm{~A}^{+} \mathrm{CD}^{+}$, and IFN- $\gamma^{+} \mathrm{IL}-17 \mathrm{~A}^{+} \mathrm{CD} 4^{+} \mathrm{T}$ cells when compared to the DSS control groups, but IL- $5^{+} \mathrm{CD} 4^{+} \mathrm{T}$ cells were not detected in all the groups (data not shown). Finally, we analyzed regulatory $\mathrm{T}$ cells, which can suppress activated T cells, in all the groups. Surprisingly, the 


\section{Cellular Physiology Cell Physiol Biochem 2019;52:1117-1138 \\ \begin{tabular}{ll|l} 
and Biochemistry $\begin{array}{l}\text { DOl: 10.33594/000000076 } \\
\text { Published online: } 17 \text { April 2019 }\end{array}$ & $\begin{array}{l}\text { O } 2019 \text { The Author(s). Published by } \\
\text { Cell Physiol Biochem Press GmbH\&Co. KG }\end{array}$ \\
\cline { 2 - 3 } &
\end{tabular} \\ Kim et al.: Gamma-Irradiated Resveratrol Induces Tolerance and Anti-Inflammatory \\ Activity in Dendritic Cells}

$\gamma$-Res-treated DSS-induced groups had dramatically increased levels of Foxp $3^{+}$regulatory $\mathrm{T}$ cells when compared to the DSS control group (Fig. 12B). These results indicate that the decreased T cell activation and increased regulatory T cells induced by the $\gamma$-Res treatment may contribute to the protective effect against DSS-induced colitis.

\section{Discussion}

Resveratrol, which is an anti-inflammatory component, ameliorates symptoms of inflammatory diseases, such as inflammatory bowel disease (IBD), rheumatoid arthritis, and systemic lupus erythematous, although it causes cell death when used in high concentrations $[13,26]$. The results of this study demonstrated that the structurally changed compound, $\gamma$-Res, of resveratrol via gamma irradiation, which is not toxic to dendritic cells (DCs) for a high-dose treatment, has immunosuppressive properties; it induces the generation of tolerogenic DCs with decreased surface molecule expression (CD80, CD86, MHC-I, and MHCII), and it increases IL-10 production without altering the TNF- $\alpha$ and IL-12p70 production and anti-inflammatory activity in activated DCs, thereby promoting the inhibition of $\mathrm{T}$ cell proliferation/activation and/or the induction of regulatory $\mathrm{T}$ cells via the interaction with DCs in vitro.

The components involved in the activation/maturation and differentiation of DCs can be used as potential candidates for valuable therapeutic targets in IBD because intestinal DCs strongly express microbial receptors, and this causes rapid inflammatory responses, including $\mathrm{T}$ cell activation and polarization of Th1, Th2, and Th17, mediated by the overexpression of surface molecules, cytokines, and chemokines [27, 28]. Therefore, if particular components inhibit the inflammatory response of DCs, it would be a promising treatment. Taking into account the inflammation, a recent study showed that blocking treatments for TNF- $\alpha$, such as by using etanercept and adalimumab, induced excessive inflammation of IBD, translating into changes in the DC function. These treatments were shown to downregulate the expression of surface molecules, such as CD83, CD80, and CD86, on DCs and to reduce their T cell stimulatory capacity [29] Recently, granulocyte-colony stimulating factor (G-CSF), which is a proliferation, differentiation, and activation factor for hematopoietic cells, treatment in Crohn's disease patients showed a therapeutic effect via reduction of IFN- $\gamma$-producing $\mathrm{T}$ cells and induction of IL-10-producing and Foxp3 $3^{+}$-expressing $\mathrm{CD} 4^{+} \mathrm{T}$ cells [30]. The function of G-CSF as a treatment was recently reported as inducing tolerogenic phenotypes during DC differentiation, and the DCs also polarize naïve $\mathrm{T}$ cells to a regulatory $\mathrm{T}$ celllike functional profile $[31,32]$. Here, regulatory $\mathrm{T}$ cells $\left(\right.$ Foxp $3^{+} \mathrm{CD} 4^{+} \mathrm{T}$ cells) have a role in immune regulation, such as inhibiting inflammation; however, reductions of these cells are associated with IBD pathogenesis [33]. The immune-modulating components involved in tolerogenic properties and anti-inflammatory activity during DC activation/differentiation may therefore serve as good candidates for IBD treatment according to their regulation of excessive inflammatory responses and induction of immune tolerance. We used $\gamma$-Res as a treatment for the DSS-induced colitis because it contributes to the generation of tolerogenic DCs and anti-inflammatory effects.

Recently, inhibitors of both MAPK and NF- $\kappa$ B signaling, which are key regulators of an excessive inflammatory response, and the proteins associated with the signaling have been evaluated due to their potential application in the treatment for IBD [34-36]. Takada et al. reported that Fos proteins and Bay 11-7085 are inhibitors of NF- $\mathrm{BB}$ signals and induce less severe clinical score and lower pro-inflammatory cytokine production in mice susceptible to DSS-induced colitis [34]. Among the MAPK inhibitor treatments, SB203580, which is a p38 inhibitor, also reduces disease activity, histological disease score, and pro-inflammatory cytokines in a DSS-induced colitis model [35]. In addition, a STAT3 inhibitor, subject to phosphorylation by JNK1/2, has been evaluated with respect to T-cell-mediated intestinal inflammation in CD patients; this treatment reduced T-cell-mediated intestinal inflammation [36]. 


\section{Cellular Physiology Cell Physiol Biochem 2019;52:1117-1138 \begin{tabular}{l|l}
\hline DOI: 10.33594/000000076 & (c) 2019 The Author(s). Published by
\end{tabular} and BiOChemistry Published online: 17 April $2019 \quad$ Cell Physiol Biochem Press GmbH\&Co. KG \\ Activity in Dendritic Cells}

\section{Conclusion}

In conclusion, new therapeutic strategies and the development of treatments for IBD require the initiation of immune tolerance and multiple inhibitions of major inflammatory factors of IBD. Our data show that $\gamma$-Res has a critical role in anti-inflammatory activity via blocking MAPK and NF- $\mathrm{KB}$ signals in activated DCs and inducing tolerogenic DCs that are capable of both inhibiting $\mathrm{T}$ cell activation/proliferation and inducing regulatory $\mathrm{T}$ cells. Importantly, $\gamma$-Res treatment conferred protective immunity and imparted protection to DSSinduced colitis in mice. Although speculative, our in vivo results suggest that the inhibition of excessive inflammation by the $\gamma$-Res treatment is a result of blocking the MAPK and NF- $\kappa B$ signals in activated DCs and inducing tolerogenic DCs. Thus, $\gamma$-Res has the potential to be an effective treatment against IBD.

\section{Acknowledgements}

This study was supported by a National Research Foundation of Korea grant funded by the Korean Government (Grant nos. 2018M2A2B3A02072069). W. S. Kim, H.-Y. Song, and E.-B. Byun: Conceived and designed the experiments; W. S. Kim, H.-Y. Song, S. Mushtag, J.-M. Kim, J.-M. Yuk, and E.-H. Byun: Performed the experiments; W. S. Kim, S. Mushtag, J.-M. Kim, J.-M. Yuk, and E.-H. Byun: Contributed reagents/materials/analysis tools; W. S. Kim, and E.-B. Byun: Wrote the paper.

\section{Disclosure Statement}

No conflicts of interest exist.

\section{References}

1 Matricon J, Barnich N, Ardid D: Immunopathogenesis of inflammatory bowel disease. Self Nonself 2010;1:299-309.

2 Hevia A, Lopez P, Suarez A, Jacquot C, Urdaci MC, Margolles A, Sanchez B: Association of levels of antibodies from patients with inflammatory bowel disease with extracellular proteins of food and probiotic bacteria. Biomed Res Int 2014;2014:351204.

- 3 Kakazu T, Hara J, Matsumoto T, Nakamura S, Oshitani N, Arakawa T, Kitano A, Nakatani K, Kinjo F, Kuroki T: Type 1 T-helper cell predominance in granulomas of Crohn's disease. Am J Gastroenterol 1999;94:21492155.

-4 Beltran CJ, Candia E, Erranz B, Figueroa C, Gonzalez MJ, Quera R, Hermoso MA: Peripheral cytokine profile in Chilean patients with Crohn's disease and ulcerative colitis. Eur Cytokine Netw 2009;20:33-38.

-5 Silva FA, Rodrigues BL, Ayrizono ML, Leal RF: The Immunological Basis of Inflammatory Bowel Disease. Gastroenterol Res Pract 2016;2016:2097274.

-6 Muzes G, Molnar B, Tulassay Z, Sipos F: Changes of the cytokine profile in inflammatory bowel diseases. World J Gastroenterol 2012;18:5848-5861.

- 7 Strober W, Fuss IJ: Proinflammatory cytokines in the pathogenesis of inflammatory bowel diseases. Gastroenterology 2011;140:1756-1767.

- 8 Mazzon E, Muia C, Paola RD, Genovese T, Menegazzi M, De Sarro A, Suzuki H, Cuzzocrea S: Green tea polyphenol extract attenuates colon injury induced by experimental colitis. Free Radic Res 2005;39:10171025.

-9 Varilek GW, Yang F, Lee EY, deVilliers WJ, Zhong J, Oz HS, Westberry KF, McClain CJ: Green tea polyphenol extract attenuates inflammation in interleukin-2-deficient mice, a model of autoimmunity. J Nutr 2001;131:2034-2039. 


\section{Cellular Physiology Cell Physiol Biochem 2019;52:1117-1138 \begin{tabular}{l|l}
\hline DOI: 10.33594/000000076 & (c)19 The Author(s). Published by
\end{tabular} and Biochemistry Published online: 17 April $2019 \quad$ Cell Physiol Biochem Press GmbH\&Co. KG \\ Kim et al.: Gamma-Irradiated Resveratrol Induces Tolerance and Anti-Inflammatory \\ Activity in Dendritic Cells}

-10 Zhang M, Deng C, Zheng J, Xia J, Sheng D: Curcumin inhibits trinitrobenzene sulphonic acid-induced colitis in rats by activation of peroxisome proliferator-activated receptor gamma. Int Immunopharmacol 2006;6:1233-1242.

-11 Shapiro H, Singer P, Halpern Z, Bruck R: Polyphenols in the treatment of inflammatory bowel disease and acute pancreatitis. Gut 2007;56:426-435.

-12 Matsuo M, Sasaki N, Saga K, Kaneko T: Cytotoxicity of flavonoids toward cultured normal human cells. Biol Pharm Bull 2005;28:253-259.

13 Mukherjee S, Dudley JI, Das DK: Dose-dependency of resveratrol in providing health benefits. Dose Response 2010;8:478-500.

14 Lu LY, Ou N, Lu QB: Antioxidant induces DNA damage, cell death and mutagenicity in human lung and skin normal cells. Sci Rep 2013;3:3169.

-15 Badaboina S, Bai HW, Na YH, Park CH, Kim TH, Lee TH, Chung BY: Novel Radiolytic Rotenone Derivative, Rotenoisin B with Potent Anti-Carcinogenic Activity in Hepatic Cancer Cells. Int J Mol Sci 2015;16:1680616815.

16 Byun EB, Song HY, Mushtaq S, Kim HM, Kang JA, Yang MS, Sung NY, Jang BS, Byun EH: Gamma-Irradiated Luteolin Inhibits 3-Isobutyl-1-Methylxanthine-Induced Melanogenesis Through the Regulation of CREB/ MITF, PI3K/Akt, and ERK Pathways in B16BL6 Melanoma Cells. J Med Food 2017;20:812-819.

17 Wang J, Pearse DD: Therapeutic Hypothermia in Spinal Cord Injury: The Status of Its Use and Open Questions. Int J Mol Sci 2015;16:16848-16879.

- 18 Kim JS, Kim WS, Choi HG, Jang B, Lee K, Park JH, Kim HJ, Cho SN, Shin SJ: Mycobacterium tuberculosis RpfB drives Th1-type T cell immunity via a TLR4-dependent activation of dendritic cells. J Leukoc Biol 2013;94:733-749.

19 Park HJ, Lee SW, Kwon DJ, Heo SI, Park SH, Kim SY, Hong S: Oral administration of taheebo (Tabebuia avellanedae Lorentz ex Griseb.) water extract prevents DSS-induced colitis in mice by up-regulating type II T helper immune responses. BMC Complement Altern Med 2017;17:448.

20 Byun EB, Kim HM, Sung NY, Yang MS, Kim WS, Choi D, Mushtaq S, Lee SS, Byun EH: Gamma irradiation of aloe-emodin induced structural modification and apoptosis through a ROS- and caspase-dependent mitochondrial pathway in stomach tumor cells. Int J Radiat Biol 2018;94:403-416.

-21 Raker VK, Domogalla MP, Steinbrink K: Tolerogenic Dendritic Cells for Regulatory T Cell Induction in Man. Front Immunol 2015;6:569.

- 22 Torres-Aguilar H, Aguilar-Ruiz SR, Gonzalez-Perez G, Munguia R, Bajana S, Meraz-Rios MA, Sanchez-Torres C: Tolerogenic dendritic cells generated with different immunosuppressive cytokines induce antigenspecific anergy and regulatory properties in memory CD4+ T cells. J Immunol 2010;184:1765-1775.

23 Zhang G, Ghosh S: Toll-like receptor-mediated NF-kappaB activation: a phylogenetically conserved paradigm in innate immunity. J Clin Invest 2001;107:13-19.

24 Corinti S, Albanesi C, la Sala A, Pastore S, Girolomoni G: Regulatory activity of autocrine IL-10 on dendritic cell functions. J Immunol 2001;166:4312-4318.

-25 Larmonier CB, Shehab KW, Ghishan FK, Kiela PR: T Lymphocyte Dynamics in Inflammatory Bowel Diseases: Role of the Microbiome. Biomed Res Int 2015;2015:504638.

26 Oliveira ALB, Monteiro VVS, Navegantes-Lima KC, Reis JF, Gomes RS, Rodrigues DVS, Gaspar SLF, Monteiro MC: Resveratrol Role in Autoimmune Disease-A Mini-Review. Nutrients 2017;9:piiE1306.

27 Bates J, Diehl L: Dendritic cells in IBD pathogenesis: an area of therapeutic opportunity? J Pathol 2014;232:112-120.

28 Steinbach EC, Plevy SE: The role of macrophages and dendritic cells in the initiation of inflammation in IBD. Inflamm Bowel Dis 2014;20:166-175.

29 Baldwin HM, Ito-Ihara T, Isaacs JD, Hilkens CM: Tumour necrosis factor alpha blockade impairs dendritic cell survival and function in rheumatoid arthritis. Ann Rheum Dis 2010;69:1200-1207.

-30 Mannon PJ, Leon F, Fuss IJ, Walter BA, Begnami M, Quezado M, Yang Z, Yi C, Groden C, Friend J, Hornung RL, Brown M, Gurprasad S, Kelsall B, Strober W: Successful granulocyte-colony stimulating factor treatment of Crohn's disease is associated with the appearance of circulating interleukin-10-producing T cells and increased lamina propria plasmacytoid dendritic cells. Clin Exp Immunol 2009;155:447-456.

31 Arpinati M, Green CL, Heimfeld S, Heuser JE, Anasetti C: Granulocyte-colony stimulating factor mobilizes T helper 2-inducing dendritic cells. Blood 2000;95:2484-2490. 


\section{Cellular Physiology and Biochemistry}

Cell Physiol Biochem 2019;52:1117-1138

Activity in Dendritic Cells

32 Rutella S, Pierelli L, Bonanno G, Sica S, Ameglio F, Capoluongo E, Mariotti A, Scambia G, d'Onofrio G, Leone G: Role for granulocyte colony-stimulating factor in the generation of human T regulatory type 1 cells. Blood 2002;100:2562-2571.

-33 Boden EK, Snapper SB: Regulatory T cells in inflammatory bowel disease. Curr Opin Gastroenterol 2008;24:733-741.

34 Takada Y, Ray N, Ikeda E, Kawaguchi T, Kuwahara M, Wagner EF, Matsuo K: Fos proteins suppress dextran sulfate sodium-induced colitis through inhibition of NF-kappaB. J Immunol 2010;184:1014-1021.

35 Hollenbach E, Neumann M, Vieth M, Roessner A, Malfertheiner P, Naumann M: Inhibition of p38 MAP kinase- and RICK/NF-kappaB-signaling suppresses inflammatory bowel disease. FASEB J 2004;18:15501552.

-36 Atreya R, Mudter J, Finotto S, Mullberg J, Jostock T, Wirtz S, Schutz M, Bartsch B, Holtmann M, Becker C, Strand D, Czaja J, Schlaak JF, Lehr HA, Autschbach F, Schurmann G, Nishimoto N, Yoshizaki K, Ito H, Kishimoto T, Galle PR, Rose-John S, Neurath MF: Blockade of interleukin 6 trans signaling suppresses T-cell resistance against apoptosis in chronic intestinal inflammation: evidence in crohn disease and experimental colitis in vivo. Nat Med 2000;6:583-588. 